\title{
On the 3D Cahn-Hilliard equation with inertial term
}

\author{
Maurizio Grasselli, Giulio Schimperna, Antonio Segatti And \\ SERGEY ZELIK
}

\begin{abstract}
We study the modified Cahn-Hilliard equation proposed by Galenko et al. in order to account for rapid spinodal decomposition in certain glasses. This equation contains, as additional term, the second-order time derivative of the (relative) concentration multiplied by a (small) positive coefficient $\varepsilon$. Thus, in absence of viscosity effects, we are in presence of a Petrovsky type equation and the solutions do not regularize in finite time. Many results are known in one spatial dimension. However, even in two spatial dimensions, the problem of finding a unique solution satisfying given initial and boundary conditions is far from being trivial. A fairly complete analysis of the 2D case has been recently carried out by Grasselli, Schimperna and Zelik. The 3D case is still rather poorly understood but for the existence of energy bounded solutions. Taking advantage of this fact, Segatti has investigated the asymptotic behavior of a generalized dynamical system which can be associated with the equation. Here we take a step further by establishing the existence and uniqueness of a global weak solution, provided that $\varepsilon$ is small enough. More precisely, we show that there exists $\varepsilon_{0}>0$ such that well-posedness holds if (suitable) norms of the initial data are bounded by a positive function of $\varepsilon \in\left(0, \varepsilon_{0}\right)$ which goes to $+\infty$ as $\varepsilon$ tends to 0 . This result allows us to construct a semigroup $S_{\varepsilon}(t)$ on an appropriate (bounded) phase space and, besides, to prove the existence of a global attractor. Finally, we show a regularity result for the attractor by using a decomposition method and we discuss the existence of an exponential attractor.
\end{abstract}

\section{Introduction}

This paper is devoted to the analysis of the following modified Cahn-Hilliard equation

$$
\varepsilon u_{t t}+u_{t}-\Delta(-\Delta u+f(u))=g,
$$

on $\Omega \times(0,+\infty), \Omega$ being a bounded smooth subset of $\mathbb{R}^{3}$, endowed with initial and boundary conditions

$$
\left\{\begin{array}{l}
\left.u\right|_{t=0}=u_{0},\left.\quad u_{t}\right|_{t=0}=u_{1}, \\
\left.u\right|_{\partial \Omega}=\left.\Delta u\right|_{\partial \Omega}=0
\end{array}\right.
$$

Here $\varepsilon>0, f$ is the derivative of a nonconvex potential (e.g., $f$ is a polynomial of degree $2 n+1, n \in \mathbb{N}$, with positive leading coefficient) and $g$ is a given (time-independent) function. In the case $\varepsilon=0$, Eq. (1.1) reduces to the well-known

Mathematics Subject Classification (2000): 35B40, 35B41, 82C26

Keywords: Cahn-Hilliard equation, Weak solutions, Global existence, Global attractors, Exponential attractors. 
Cahn-Hilliard equation (cf. [5]) which has been widely analyzed by many authors (see, e.g., [8,9,22,24-28,31,35] and references therein). We recall that this equation describes the phase separation of a binary mixture and $u$ denotes the relative concentration of one species. This kind of phenomenon is rather complex and one of its typical features is the so-called spinodal decomposition (see, for instance, $[4,18,23])$. To model non-equilibrium decompositions caused by deep supercooling in certain glasses, Galenko et al. (cf. [11-13]) have proposed to modify the usual CahnHilliard equation by adding the inertial term $\varepsilon u_{t t}$. This equation shows a good agreement with experiments performed on glasses (see [14]). It is worth remarking that, from the practical viewpoint, the parameter $\varepsilon$ is usually very small with respect to the other physical constants, which have been all set equal to one for simplicity (see, however, Remark 2.8).

Equation (1.1) endowed, e.g., with conditions (1.2), has different mathematical features with respect to the Cahn-Hilliard equation since it is no longer parabolic and solutions do not get smoother in finite time. Therefore, its analysis presents some difficulties which can be overcome rather easily in one dimension. This is due to the fact that energy bounded solutions (i.e., of $H^{1} \times\left(H^{1}\right)^{\prime}$-type, cf. Definition 2.1 below) are also globally bounded thanks to the injection $H^{1}(\Omega) \hookrightarrow L^{\infty}(\Omega)$. For the same reason, uniqueness of energy bounded solutions holds for potentials of any growth. As a consequence, there is a number of results concerning the one-dimensional case (see $[7,15,17,36,37])$. The two-dimensional case is much more complicated also because energy bounded solutions are no longer bounded in $L^{\infty}(\Omega)$. This case has been treated only recently in [20]. Actually, in dimension two, the existence of an energy bounded solution is relatively easy to prove, but its uniqueness is far less standard and was obtained in [20] for potentials growing (at most) like a polynomial of degree 4. In that work it was also shown that a dynamical system can be defined on the phase space of the energy bounded solutions and it possesses a global attractor. A further similar result was established in [20] for quasi-strong solutions, that is, originating from a phase space of $H^{3} \times H^{1}$-type. One of the key tools for proving these two-dimensional results is a logarithmic interpolation inequality which holds in dimension two only (see [3]) and whose use forces the restriction on the growth of the potential.

Thus, the three-dimensional case must be analyzed by means of a different approach. As far as we know, the only available results so far are contained in [32], where the author adopts the approach of multi-valued semigroups and related (generalized) global attractor developed by Babin and Vishik (cf. [1], see also [2]) to Eq. (1.1). This approach is based solely on the existence of energy bounded solutions (actually the mere existence property is still easy to show for energy solutions, also in dimension three). Other authors have studied this equation by adding a viscous term like $-\Delta u_{t}$. In this case solutions regularize in finite time and, consequently, the corresponding mathematical analysis becomes simpler, though nontrivial (see [6,16,21], cf. also [34] where smoothing properties are due to weakly singular memory kernels). 
As observed in [20], Eq. (1.1) bears some similarities with the semilinear damped wave equation. However, in contrast to that case, for Eq. (1.1) in dimension three, any non-quadratic potential appears to show a "supercritical" character. In particular, energy solutions could be in some sense too weak and possibly non-unique, whereas "stronger" solutions should have better properties, of course, provided they exist globally in time. Thus, it seemed natural to try to extend the approach to the supercritical damped wave equations developed in [38] (see also [19,39]) to Eq. (1.1). Recall that, roughly speaking, this approach consists of two key steps. First, one needs to verify the global well-posedness and dissipativity of smooth solutions, provided that $\varepsilon$ is small enough and the initial data are suitably bounded (in a stronger energy space) by a constant $R_{\varepsilon}$ which blows up as $\varepsilon$ goes to 0 . The second (and probably more delicate) step consists in showing that any energy solution is asymptotically smooth and tends to the global attractor for the smooth solutions whose existence has been previously obtained.

In the present paper we focus on the first step of the above mentioned strategy, namely, on the global existence and uniqueness of sufficiently regular solutions with initial energy bounded by $R_{\varepsilon}$. The second step, related with the energy solutions, will be considered in a forthcoming paper. Therefore, we begin to establish the global existence and dissipativity of the $H^{2} \times L^{2}$-type solutions. These are the so-called weak solutions in our terminology and they are stronger than the $H^{1} \times H^{-1}$-type energy solutions. Then, we will show that any such solution (if it exists) is asymptotically smoother (and this regularity is restricted only by the smoothness of $f$ and $g$ ). It is also smoother from the very beginning provided that the initial data are smoother as well (even without any assumptions on the smallness of $\varepsilon$ ). Finally, we demonstrate the existence and regularity of the global attractor for the properly defined semigroup associated with Eq. (1.1) and solutions which are more regular than the energy ones. We also report the statement of a result on the existence of exponential attractors which could be shown just by following the lines of [20, Sec. 5] and whose proof is thus omitted.

Analogously to [38], the key proof of the global existence of more regular solutions for small $\varepsilon$ is based on a careful comparison of a solution with the properly chosen solution of the limiting $(\varepsilon=0)$ parabolic Cahn-Hilliard equation. However, since Eq. (1.1) is essentially more complicated than the damped wave equation, the extension of the technique of [38] to our case is far from being straightforward. In particular, even in the regular phase spaces (say, $H^{s+1} \times H^{s-1}, s>1 / 2$ ) Eq. (1.1) remains "critical" and the standard decomposition of the associated semigroup does not allow to obtain further regularity and the associated asymptotic smoothness (in contrast to the case of wave equations). We overcome this difficulty by suggesting a new decomposition of the semigroup into a compact and a decaying part, which, we believe, is of an independent interest (see (3.91) and (3.92) below). It is also worth mentioning that the boundary conditions of Navier type are chosen just for simplicity. Actually, the physically more appropriate no-flux (or periodic) boundary conditions 
could be equally considered with some additional complications due to the evolution of the spatial average of $u$ (see, e.g., [7]).

The plan of the paper is as follows. In the next section we will give our basic assumptions and state our main results. The proofs will be collected in the subsequent Sect. 3 .

\section{Mathematical formulation and results}

\subsection{Assumptions and statement of the problem}

Let us set $H:=L^{2}(\Omega)$ and denote by $(\cdot, \cdot)$ the scalar product both in $H$ and in $H \times H$, and by $\|\cdot\|$ the related norm. The symbol $\|\cdot\|_{X}$ will indicate the norm in the generic (real) Banach space $X$. Next, we set $V:=H_{0}^{1}(\Omega)$, so that $V^{\prime}=H^{-1}(\Omega)$ is the topological dual of $V$. The duality between $V^{\prime}$ and $V$ will be noted by $\langle\cdot, \cdot\rangle$. The space $V$ is endowed with the scalar product

$$
((v, z)):=\int_{\Omega} \nabla v \cdot \nabla z, \quad \forall v, z \in V,
$$

and the related norm. We shall denote by $A$ the Riesz operator on $V$ associated with (2.1), namely,

$$
A: V \rightarrow V^{\prime}, \quad\langle A v, z\rangle=((v, z))=\int_{\Omega} \nabla v \cdot \nabla z, \quad \forall v, z \in V,
$$

so that we also have the dual scalar product on $V^{\prime}$ given by

$$
((\eta, \xi))_{*}:=\left\langle\eta, A^{-1} \xi\right\rangle=\left\langle\xi, A^{-1} \eta\right\rangle, \quad \forall \xi, \eta \in V^{\prime} .
$$

Abusing notation slightly, we shall also indicate by the same letter $A$ the restriction of the operator defined in (2.2) to the set $D(A)=H^{2}(\Omega) \cap V$, i.e., the unbounded operator defined as

$$
A=-\Delta \text { with domain } D(A)=H^{2}(\Omega) \cap H_{0}^{1}(\Omega) \subset L^{2}(\Omega) .
$$

We can thus introduce the family of Hilbert spaces

$$
H^{2 s}=D\left(A^{s}\right), \quad s \in \mathbb{R},
$$

with scalar products

$$
((u, v))_{2 s}:=\left(A^{s} u, A^{s} v\right), \quad \forall u, v \in H^{s} .
$$

It is well known that $H^{s_{1}} \subset H^{s_{2}}$ with dense and compact immersion when $s_{1}>s_{2}$. For $\varepsilon \geq 0$, we introduce the scale of Hilbert spaces

$$
\mathcal{V}_{s}=\mathcal{V}_{s}(\varepsilon):= \begin{cases}D\left(A^{\frac{s+1}{2}}\right) & \text { if } \varepsilon=0 \\ D\left(A^{\frac{s+1}{2}}\right) \times D\left(A^{\frac{s-1}{2}}\right) & \text { if } \varepsilon>0\end{cases}
$$


so that we have, in particular, $\mathcal{V}_{0}(\varepsilon)=V \times V^{\prime}$ if $\varepsilon>0$ and $\mathcal{V}_{0}(0)=V$; analogously, $\mathcal{V}_{1}(\varepsilon)=\left(H^{2}(\Omega) \cap V\right) \times H$ and $\mathcal{V}_{1}(0)=H^{2}(\Omega) \cap V$. The spaces $\mathcal{V}_{s}$ are naturally endowed with the graph norm

$$
\|(u, v)\|_{s}^{2}:=\left\|A^{\frac{s+1}{2}} u\right\|^{2}+\varepsilon\left\|A^{\frac{s-1}{2}} v\right\|^{2},
$$

where the couple $(u, v)$ has to be intended as $u$ if $\varepsilon=0$.

Regarding the nonlinear function $f$, we assume that $f \in C^{2}(\mathbb{R} ; \mathbb{R})$ with $f(0)=0$ satisfies

$$
\begin{aligned}
& \liminf _{|r| \rightarrow+\infty} \frac{f(r)}{r}>-\lambda_{1} ; \\
& \exists \lambda \in[0,+\infty): f^{\prime}(r) \geq-\lambda, \quad \forall r \in \mathbb{R} .
\end{aligned}
$$

Here $\lambda_{1}$ is the first eigenvalue of $A$. Note that $f$ can be, for instance, a polynomial of arbitrarily large odd degree with positive leading coefficient. If we indicate as $F$ the potential of $f$ (i.e., a suitable primitive of $f$ ), we can always suppose that

$$
F(r) \geq-\frac{\kappa}{2} r^{2}
$$

for some $\kappa<\lambda_{1}$. By (2.8), we also have that $F$ is $\lambda$-convex. We also let

$$
g \in H \text {. }
$$

We can now introduce, for $\varepsilon>0$, the energy associated with (2.14) as

$$
\mathcal{E}_{\varepsilon}: \mathcal{V}_{0} \rightarrow \mathbb{R}, \quad \mathcal{E}_{\varepsilon}(u, v):=\frac{1}{2}\|(u, v)\|_{0}^{2}+(F(u), 1)-\left\langle g, A^{-1} u\right\rangle .
$$

Analogously, if $\varepsilon=0$, we set

$$
\mathcal{E}_{0}: V \rightarrow \mathbb{R}, \quad \mathcal{E}_{0}(u):=\frac{1}{2}\|u\|_{V}^{2}+(F(u), 1)-\left\langle g, A^{-1} u\right\rangle .
$$

Assumptions (2.7) and (2.10) guarantee that both $\mathcal{E}_{\varepsilon}$ and $\mathcal{E}_{0}$ are finite for all $(u, v) \in \mathcal{V}_{1}$. Instead, they can be $+\infty$ if $(u, v) \in \mathcal{V}_{0}$. Of course, thanks to $(2.7), \mathcal{E}_{\varepsilon}\left(\mathcal{E}_{0}\right)$ is in any case bounded from below on the whole $\mathcal{V}_{0}$ (respectively, $V$ ).

We are now ready to provide a rigorous variational reformulation of system (1.1) and (1.2). We adopt here the terminology introduced in [20].

DEFINITION 2.1. Given some reference time interval $(0, T)$, with possibly $T=$ $+\infty$, we say that a pair $\left(u, u_{t}\right)$ is an energy bounded solution, or, more concisely, energy solution, to Problem $P_{\varepsilon}$ if

$$
\left(u, u_{t}\right) \in L^{\infty}\left(0, T ; \mathcal{V}_{0}\right), \quad u_{t t} \in L^{\infty}\left(0, T, D\left(A^{-2}\right)\right), \quad \mathcal{E}_{\varepsilon}\left(u, u_{t}\right) \in L^{\infty}(0, T),
$$

and the following relations hold

$$
\begin{aligned}
& A^{-1}\left(\varepsilon u_{t t}+U_{t}\right)+A u+f(u)=A^{-1} g, \quad \text { in } D\left(A^{-1}\right), \text { for a.e. } t \in(0, T), \\
& \left.u\right|_{t=0}=u_{0}, \quad \text { in } V,\left.\quad u_{t}\right|_{t=0}=u_{1}, \quad \text { in } V^{\prime} .
\end{aligned}
$$


REMARK 2.2. The above definition requires a number of comments. Firstly, we observe that the regularity condition (2.13) is sufficient to ensure that all single term in (2.14) belong to $D\left(A^{-1}\right)$ for almost any values of the time variable. Indeed, the regularity of $\mathcal{E}$ guarantees in particular that, almost everywhere in $(0, T), f(u) \in$ $L^{1}(\Omega) \subset D\left(A^{-1}\right)$. Concerning the initial conditions, they make sense in the spaces specified in (2.15) since the regularity of $u_{t}$ and $u_{t t}$ in (2.13) ensures that

$$
u \in C_{w}^{0}([0, T] ; V), \quad u_{t} \in C_{w}^{0}\left([0, T], V^{\prime}\right),
$$

where we recall that $C_{w}^{0}([0, T] ; X)$ is defined as ( $X$ being a real Banach space)

$$
C_{w}^{0}([0, T] ; X):=\left\{v \in L^{\infty}(0, T ; X):\langle\phi, v(\cdot)\rangle \in C^{0}([0, T]), \quad \forall \phi \in X^{\prime}\right\} .
$$

In the sequel we will frequently write $U$ for the couple $\left(u, u_{t}\right)$ and $U_{0}$ for $\left(u_{0}, u_{1}\right)$ (the same convention will be kept for other letters, e.g., we will write $V=\left(v, v_{t}\right)$ ). Moreover, for the sake of brevity, solutions will be sometimes denoted simply as $u$, or as $U$, rather than as $\left(u, u_{t}\right)$. We can now introduce three stronger notions of solution.

DEFINITION 2.3. Given some $(0, T)$ as in Definition 2.1, we say that an energy solution $U$ to Problem $P_{\varepsilon}$ is a weak solution if we have

$$
\left(u, u_{t}\right) \in L^{\infty}\left(0, T ; \mathcal{V}_{1}\right), \quad u_{t t} \in L^{\infty}\left(0, T, D\left(A^{-1}\right)\right) .
$$

Moreover, an energy solution $U$ to Problem $P_{\varepsilon}$ is a quasi-strong solution if we have

$$
\left(u, u_{t}\right) \in L^{\infty}\left(0, T ; \mathcal{V}_{2}\right), \quad u_{t t} \in L^{\infty}\left(0, T, V^{\prime}\right),
$$

while $U$ is a strong solution if we have

$$
\left(u, u_{t}\right) \in L^{\infty}\left(0, T ; \mathcal{V}_{3}\right), \quad u_{t t} \in L^{\infty}(0, T, H)
$$

Of course, for weak, quasi-strong and strong solutions Eq. (2.14) and initial conditions (2.15) can correspondingly be interpreted in a stronger sense.

REMARK 2.4. Notice that, despite the name, energy bounded solutions are weaker than weak solutions. Observe also that, in some sense, there is a larger regularity gap between energy and weak solutions than between weak and quasi-strong solutions. Indeed, the regularity of energy solutions is not sufficient to ensure that $f(u)$ belongs to $V^{\prime}$ (which would also allow to read (2.14) in $V^{\prime}$ instead that in $D\left(A^{-1}\right)$ ). Thus, the non-linearity $\Delta f(u)$ is not subordinated to the linear part of the equation and, for this reason, Eq. (1.1) is "supercritical" in the class of energy solutions. In contrast to that, thanks to the embedding $H^{s+1}(\Omega) \hookrightarrow C(\bar{\Omega}), s>1 / 2$, the assumption $u \in H^{s+1}(\Omega)$ implies that $\Delta f(u) \in H^{s-1}(\Omega)$ (but, nevertheless, it does not belong to $H^{s-1+\delta}(\Omega)$ for any positive $\delta$ ) if $s>1 / 2$. Therefore, Eq. (1.1) becomes "critical" in any phase space $H^{s+1}(\Omega) \times H^{s}(\Omega)$ for $s>1 / 2$ and, in particular, in the case of weak solutions. That explains why the asymptotic smoothing property is nontrivial even in the case of regular solutions (in contrast to the usual wave equation which becomes "subcritical" in the classes of regular solutions). 


\subsection{Local and global well-posedness}

As noticed in the Introduction, although it is relatively easy to show existence of energy solutions to Problem $P_{\varepsilon}$ under the natural growth restrictions on the potential, here we will be interested only in the (more restrictive) class of weak solutions for which we start proving a local in time existence result.

THEOREM 2.5. Let the assumptions (2.7), (2.8) and (2.10) hold. If

$$
U_{0}=\left(u_{0}, u_{1}\right) \in \mathcal{V}_{1},
$$

then there exist a time $T_{0}=T_{0}\left(\left\|U_{0}\right\|_{1},\|g\|\right)>0$ and a constant $\mathcal{Q}=\mathcal{Q}\left(\left\|U_{0}\right\|_{1},\|g\|\right)>$ 0 both independent of $\varepsilon$, such that Problem $P_{\varepsilon}$ has a solution $U=\left(u, u_{t}\right)$ in $\left[0, T_{0}\right]$ which satisfies

$$
\|U(t)\|_{1} \leq \mathcal{Q}\left(\left\|U_{0}\right\|_{1},\|g\|\right), \quad \forall t \in\left[0, T_{0}\right]
$$

Taking advantage of the additional regularity, we also have uniqueness.

THEOREM 2.6. Let the assumptions of Theorem 2.5 hold and let $\left(u^{1}, u_{t}^{1}\right)$ and $\left(u^{2}, u_{t}^{2}\right)$ be a pair of weak solutions defined on some interval $(0, T)$ and having the same initial data $\left(u_{0}, u_{1}\right)$ with the regularity (2.20). Then $\left(u^{1}, u_{t}^{1}\right)$ and $\left(u^{2}, u_{t}^{2}\right)$ do coincide on $(0, T)$.

Next, we pass to our main result. This states that, under a restriction on the magnitude of the initial data, the local solution to $P_{\varepsilon}$ can be extended to a global one (which is still unique thanks to Theorem 2.6).

THEOREM 2.7. Let the assumptions of Theorem 2.5 hold. Then, there exist $\varepsilon_{0}>0$ and a nonincreasing positive function $R:\left(0, \varepsilon_{0}\right) \rightarrow(0,+\infty)$ with the property

$$
\lim _{\varepsilon \searrow 0} R(\varepsilon)=+\infty
$$

such that, for every $\varepsilon \in\left(0, \varepsilon_{0}\right)$ and every initial condition $U_{0}=\left(u_{0}, u_{1}\right) \in \mathcal{V}_{1}$ satisfying

$$
\left\|U_{0}\right\|_{1} \leq R(\varepsilon)
$$

there exists a (unique) global weak solution $U=\left(u, u_{t}\right)$ to problem $P_{\varepsilon}$ such that

$$
\|U(t)\|_{1} \leq Q\left(\left\|U_{0}\right\|_{1}\right) e^{-\kappa t}+Q(\|g\|), \quad \forall t \geq 0,
$$

for some positive constant $\kappa$ and some positive increasing monotone function $Q$ both independent of $\varepsilon$. Moreover, $u$ satisfies the energy dissipation inequality

$$
\|U(t)\|_{0}^{2}+\int_{0}^{t} e^{-\kappa(t-s)}\left\|u_{t}(s)\right\|_{V^{\prime}}^{2} \mathrm{~d} s \leq Q\left(\left\|U_{0}\right\|_{1}^{2}\right) e^{-\kappa t}+Q\left(\|g\|^{2}\right), \quad \forall t \geq 0,
$$


where the positive increasing monotone function $Q$ and the positive constant $\kappa$ are independent of $\varepsilon$, as well as the higher order identity

$$
\begin{aligned}
& I(U)(M) \\
& =\mathrm{e}^{-\frac{M}{\varepsilon}} I\left(U_{0}\right)+\int_{0}^{M} \mathrm{e}^{\frac{t-M}{\varepsilon}}\left[\left(g, u_{t}+\frac{1}{2 \varepsilon} u\right)+\frac{1}{2}\left(f^{\prime \prime}(u)|\nabla u|^{2}, u_{t}\right)\right] \mathrm{d} t, \\
& \quad \forall M>0,
\end{aligned}
$$

where $I=I(U)$ is defined by

$$
I(U):=\frac{\varepsilon}{2}\left\|u_{t}\right\|^{2}+\frac{1}{2}\|A u\|^{2}+\frac{1}{2}\left(u_{t}, u\right)+\frac{1}{2}\left(f^{\prime}(u) \nabla u, \nabla u\right) .
$$

REMARK 2.8. On account of [38, Rem. 3.4], we can observe that if we consider

$$
\sigma u_{t t}+\gamma u_{t}-\Delta(-\Delta u+f(u))=g,
$$

where $\sigma, \gamma>0$, then, by rescaling the equation with $t \mapsto \gamma t^{\prime}$, we obtain Eq. (1.1) with $\varepsilon=\frac{\sigma}{\gamma^{2}}$. Therefore, the constraint on $\varepsilon$ is related with both the inertial and the damping coefficients.

For the reader's convenience, we also recall a result regarding the parabolic case, whose proof is standard (see, e.g., [8,22,33] for details).

THEOREM 2.9. Let (2.7), (2.8) and (2.10) hold and let $T>0$. If $u_{0} \in V$ is such that $\mathcal{E}_{0}\left(u_{0}\right)<\infty$ (hence, in particular, if $u_{0} \in D(A)$ ), then there exists a unique function

$$
u \in H^{1}\left(0, T ; V^{\prime}\right) \cap L^{\infty}(0, T ; V) \cap L^{2}(0, T ; D(A))
$$

which solves

$$
A^{-1} u_{t}+A u+f(u)=A^{-1} g, \quad \text { in } H, \text { a.e. in }(0, T) .
$$

\subsection{Long time behavior}

Theorem 2.7 allows us to interpret $P_{\varepsilon}$ as a dynamical system defined on a suitable phase space. Indeed, for $\varepsilon \in\left(0, \varepsilon_{0}\right)$, let us set

$$
B_{\varepsilon}(R):=\left\{U_{0}=\left(u_{0}, u_{1}\right) \in \mathcal{V}_{1}:\left\|U_{0}\right\|_{1} \leq R\right\},
$$

where $R=R(\varepsilon)$ is as in Theorem 2.7. Thanks to this theorem, we have that

$$
S_{\varepsilon}(t) U_{0}:=U(t)=\left(u(t), u_{t}(t)\right), \quad \forall U_{0} \in B_{\varepsilon}(R), \forall t \geq 0,
$$

is a semigroup. We now define

$$
\mathcal{B}_{\varepsilon}:=\left[\bigcup_{t \geq 0} S_{\varepsilon}(t) B_{\varepsilon}(R)\right]_{\mathcal{V}_{1}},
$$


where $[\cdot] \mathcal{V}_{1}$ denotes the closure with respect to the strong topology of $\mathcal{V}_{1}$. Owing to estimate (2.24), $\mathcal{B}_{\varepsilon}$ is a bounded and, of course, closed set of $\mathcal{V}_{1}$ which is invariant under the action of $S_{\varepsilon}(t)$. Therefore, thanks to (2.24), $\left(\mathcal{B}_{\varepsilon}, S_{\varepsilon}(t)\right)$ is a (dissipative) dynamical system. We now come to our first result.

THEOREM 2.10. Let the assumptions of Theorem 2.7 hold. Then, for all $\varepsilon \in\left(0, \varepsilon_{0}\right)$ there exists a compact global attractor $\mathcal{A}_{\varepsilon} \subset \mathcal{V}_{1}$ for the semigroup $S_{\varepsilon}(t)$ on the phase space $\mathcal{B}_{\varepsilon}$ which is bounded in $\mathcal{V}_{2}$. Moreover, if $f \in C_{\text {loc }}^{2,1}(\mathbb{R} ; \mathbb{R})$, then there exists $\varepsilon_{1} \in\left(0, \varepsilon_{0}\right]$ such that, for all $\varepsilon \in\left(0, \varepsilon_{1}\right), \mathcal{A}_{\varepsilon}$ is bounded in $\mathcal{V}_{3}$.

REMARK 2.11. Being $\mathcal{B}_{\varepsilon}$ bounded, the attraction property can be formulated simply as

$$
\lim _{t \rightarrow+\infty} \operatorname{dist}_{1}\left(S_{\varepsilon}(t) \mathcal{B}_{\varepsilon}, \mathcal{A}_{\varepsilon}\right)=0
$$

where dist ${ }_{1}$ denotes the Hausdorff semidistance of sets in the norm of $\mathcal{V}_{1}$.

REMARK 2.12. The main issue in the above Theorem is the $\mathcal{V}_{3}$-regularity of the attractor. Actually, the existence of a global attractor bounded in $\mathcal{V}_{1}$ could be achieved in a relatively standard way by using, for instance, the equality (2.26) together with the so-called energy method by Ball (cf. [2]). To show that the attractor has the $\mathcal{V}_{3}$ regularity, we will proceed in two steps: first we will show a $\mathcal{V}_{1}-\mathcal{V}_{2}$ regularization estimate providing the existence of a $\mathcal{V}_{2}$-bounded exponentially attracting set (see Sect. 3.4). This is enough to ensure the existence of the global attractor bounded in $\mathcal{V}_{2}$. Then we achieve a $\mathcal{V}_{2}-\mathcal{V}_{3}$ smoothing property by means of a decomposition procedure similar to that used for the two-dimensional problem in [20] (see the following Sect. 3.5). However, to complete the proof we need to use the transitivity property of exponential attraction (see [10, Thm. 5.1]) to guarantee that $\mathcal{A}_{\varepsilon}$ actually attracts also solutions with initial data in $\mathcal{V}_{1}$. This property holds provided that $S_{\varepsilon}(t)$ is Lipschitz continuous on $\mathcal{V}_{1}$ and, in order to prove that, we must require $f \in C_{\text {loc }}^{2,1}(\mathbb{R} ; \mathbb{R})$, namely, we also need $f^{\prime \prime}$ to be locally Lipschitz continuous (see Lemma 3.8 below). Moreover, this assumption is needed for proving the $\mathcal{V}_{3}$-boundedness of one component of the semigroup (see (3.148)).

REMARK 2.13. We recall that the technique introduced in [38] can also be used to handle singular potentials (see [19]). Thus, with some more technicalities, the results of this paper can be proven even in the case of suitable singular potentials defined, say, on $(0,1)$ so that $u(x, t) \in(0,1)$ for all $(x, t) \in \bar{\Omega} \times(0,+\infty)$.

REMARK 2.14. Using the asymptotic compactness of the trajectories and the Łojasiewicz-Simon inequality, it could also be proved that any weak solution converges to a unique equilibrium, whenever $f$ is real analytic.

Finally, we move to our last result, dealing with the exponential attractor issue. We will not prove this result, since the argument would follow with almost no modifications the proof of [20, Thm. 5.1]. The only significant difference is that here we have 
to take care of the further constraint (2.23), but this affects just the statement and not the proof.

THEOREM 2.15. Let the assumptions of Theorem 2.5 together with (2.23) hold. In addition, suppose that $f \in C_{\text {loc }}^{2,1}(\mathbb{R} ; \mathbb{R})$. Then, the semigroup $\mathcal{S}_{\varepsilon}(t)$ restricted to $\mathcal{B}_{\varepsilon}$ admits an exponential attractor $\mathcal{M}_{\varepsilon}$. Namely, $\mathcal{M}_{\varepsilon}$ is a positively invariant, compact subset of $\mathcal{V}_{1}$ with finite fractal dimension with respect to the $\mathcal{V}_{1}$-metric which is also bounded in $\mathcal{V}_{2}$ (in $\mathcal{V}_{3}$, if $\varepsilon \in\left(0, \varepsilon_{1}\right)$ ) and such that there exist positive constants $\kappa, C$ such that

$$
\operatorname{dist}_{1}\left(S_{\varepsilon}(t) \mathcal{B}_{\varepsilon}, \mathcal{M}_{\varepsilon}\right) \leq C e^{-\kappa t} \text {. }
$$

REMARK 2.16. Theorem 2.15 implies that $\mathcal{A}_{\varepsilon}$ has finite fractal dimension with respect to the $\mathcal{V}_{1}$-metric.

\section{Proofs}

In what follows, the symbols $c, \kappa$, and $c_{i}, i \geq 0$, will denote positive constants depending on the data $f, g$ of the problem, but independent of the initial datum, of time, and of $\varepsilon$. The values of $c$ and $\kappa$ are allowed to change even within a single line. Analogously, $Q: \mathbb{R}^{+} \rightarrow \mathbb{R}^{+}$(or $Q_{i}, i \geq 0$ ) will be positive increasing monotone functions defined on the whole real line (the expression of $Q$ can also vary). A monotone function noted by $q$ will be instead allowed to explode in (strictly positive) finite times. Finally, $C$, or $C_{i}, i \geq 0$, will stand for positive constants with additional dependencies (e.g., on the initial datum), specified on occurrence.

\subsection{Proof of Theorem 2.5}

The existence result relies on an approximation — a priori estimates — passage to the limit procedure. The approximation step is based on a Faedo-Galerkin scheme. The crucial point here is that the finite dimensionality of the approximating problem allows for testing (2.14) with (the discrete equivalent of) $A u_{t}$, which would not be permitted in the continuous setting.

The Faedo-Galerkin scheme is as follows. First of all, since $A^{-1}$ is compact and self-adjoint, there exists an orthonormal basis of $L^{2}(\Omega)$ consisting of eigenvectors of $A$, that is

$$
\begin{aligned}
& A v_{i}=\lambda_{i} v_{i}, \quad\left\|v_{i}\right\|=1, \text { for } i=1,2, \ldots, \\
& \left(v_{i}, v_{j}\right)=\delta_{i, j}, \quad \text { for } i \neq j, \\
& 0<\lambda_{1} \leq \lambda_{2} \leq \cdots \leq \lambda_{j} \rightarrow+\infty, \text { as } j \nearrow+\infty .
\end{aligned}
$$

It is well known that $\left\{\lambda_{j}^{-1 / 2} v_{j}\right\}_{j \geq 1}$ is an orthonormal basis of $H_{0}^{1}(\Omega)$. For any $N \geq 1$ we let $V^{N}$ be the space spanned by $\left\{v_{1}, \ldots, v_{N}\right\}$. Then we introduce the approximating problem. 
Problem $P_{N}$ : Find $t_{N}>0$ and $u_{i} \in C^{2}\left(\left[0, t_{N}\right]\right)$ for $i=1, \ldots, N$ such that, setting

$$
u^{N}:=\sum_{i=1}^{N} u_{i}(t) v_{i}
$$

then $u^{N} \in C^{2}\left(\left[0, t_{N}\right], D(A)\right)$ solves

$$
\begin{aligned}
& \varepsilon\left\langle A^{-1} u_{t t}^{N}, v\right\rangle+\left\langle A^{-1} u_{t}^{N}, v\right\rangle+\left\langle A u^{N}, v\right\rangle+\left\langle f\left(u^{N}\right), v\right\rangle \\
& \quad=\left\langle A^{-1} g^{N}, v\right\rangle \quad \forall v \in V^{N}, \\
& u^{N}(0)=u_{0}^{N}, \quad u_{t}^{N}(0)=u_{1}^{N},
\end{aligned}
$$

where $u_{0}^{N}, u_{1}^{N}$ and $g^{N}$ are suitable sequences in $V^{N}$ such that, as $N \rightarrow+\infty$,

$$
\begin{aligned}
& u_{0}^{N} \rightarrow u_{0} \quad \text { in } D(A), \\
& u_{1}^{N} \rightarrow u_{1} \quad \text { in } H, \\
& g^{N} \rightarrow g \quad \text { in } H .
\end{aligned}
$$

Problem $P_{N}$ consist of a $N$-dimensional system of nonlinear ordinary differential equations. Thus, the Cauchy-Lipschitz Theorem applies and we know that there exists a unique (maximal) solution $u^{N}$ in $\left[0, T^{*}\right), T^{*} \in(0,+\infty]$ being the life span. We now derive some a priori estimates that will lead us to prove the existence result. To this end, just for the sake of simplicity, we drop from now on the superscript $N$.

We start by deriving the standard basic energy estimate. To get it, we set $v=u_{t}$ in (3.2), so that

$$
\frac{\mathrm{d}}{\mathrm{d} t}\left[\frac{1}{2}\|U\|_{0}^{2}+(F(u), 1)-\left(g, A^{-1} u\right)\right]+\left\|u_{t}\right\|_{V^{\prime}}^{2}=0,
$$

where $U=\left(u, u_{t}\right)$. Integrating (3.7) and recalling (2.9), we deduce

$$
\|U(t)\|_{0}^{2}+\int_{0}^{t}\left\|u_{t}(s)\right\|_{V^{\prime}}^{2} \mathrm{~d} s \leq Q\left(\left\|U_{0}\right\|_{1}^{2}\right)+\|g\|^{2} .
$$

By letting $t$ go to $+\infty$ in (3.8) we obtain in particular the finiteness of the dissipation integral

$$
\int_{0}^{+\infty}\left\|u_{t}(s)\right\|_{V^{\prime}}^{2} \mathrm{~d} s \leq Q\left(\left\|U_{0}\right\|_{1}^{2}\right)+\|g\|^{2} .
$$

We now set $v=\alpha u$ in (3.2), where the constant $\alpha$ will be chosen later in a way independent of $\varepsilon$. By adding the resulting equality to (3.7), we obtain

$$
\begin{aligned}
& \frac{\mathrm{d}}{\mathrm{d} t}\left[\frac{1}{2}\|U\|_{0}^{2}+\varepsilon \alpha\left(A^{-1 / 2} u_{t}, A^{-1 / 2} u\right)+(F(u), 1)-\left(g, A^{-1} u\right)\right] \\
& \quad+(1-\varepsilon \alpha)\left\|u_{t}\right\|_{V^{\prime}}^{2}+\alpha\|\nabla u\|^{2}+\alpha\left(A^{-1 / 2} u_{t}, A^{-1 / 2} u\right)+\alpha(f(u), u) \\
& \quad-\alpha\left(g, A^{-1} u\right)=0 .
\end{aligned}
$$


Thanks to (2.8) we have that $\alpha(f(u), u) \geq-\frac{\lambda \alpha}{2}\|u\|^{2}+\alpha(F(u), 1)$. Hence, denoting by $\Phi(U)$ the quantity in square brackets in (3.10) and choosing $\alpha$ sufficiently small, we can find two positive constants $\kappa$ and $c$ such that identity (3.10) yields

$$
\frac{\mathrm{d}}{\mathrm{d} t} \Phi(U)+\kappa \Phi(U)+\kappa\left\|u_{t}\right\|_{V^{\prime}}^{2} \leq c .
$$

Thus, by making use of the standard Gronwall Lemma, we conclude that

$$
\Phi(U(t))+\int_{0}^{t} \mathrm{e}^{-\kappa(t-s)}\left\|u_{t}(s)\right\|_{V^{\prime}}^{2} \mathrm{~d} s \leq \Phi\left(U_{0}\right) \mathrm{e}^{-\kappa t}+c .
$$

Since

$$
c\|U\|_{0}^{2}-c\left(1+\|g\|^{2}\right) \leq \Phi(U) \leq Q\left(\|U\|_{1}^{2}\right)+Q\left(\|g\|^{2}\right)
$$

for some positive constant $c$, estimate (3.12) implies

$$
\begin{aligned}
& \varepsilon\left\|u_{t}(t)\right\|_{V^{\prime}}^{2}+\|\nabla u(t)\|^{2}+\int_{0}^{t} \mathrm{e}^{-\kappa(t-s)}\left\|u_{t}(s)\right\|_{V^{\prime}}^{2} \mathrm{~d} s \\
& \leq Q\left(\left\|U_{0}\right\|_{1}\right) \mathrm{e}^{-\kappa t}+Q(\|g\|), \quad \forall t \geq 0,
\end{aligned}
$$

for some positive constant $\kappa$ independent of $\varepsilon$. Thus, (2.25) follows as well. We set now $v=A\left(u_{t}+\alpha u\right)$ in (3.2), $\alpha$ being as before a positive parameter to be chosen later on. We thus obtain

$$
\begin{aligned}
& \frac{\mathrm{d}}{\mathrm{d} t}\left[\frac{\varepsilon}{2}\left\|u_{t}\right\|^{2}+\frac{1}{2}\|A u\|^{2}-(g, u)\right]+\varepsilon \alpha\left\langle u_{t t}, u\right\rangle+\alpha\left(u_{t}, u\right)+\left\|u_{t}\right\|^{2}+\alpha\|A u\|^{2} \\
& \quad+\left(A f(u), u_{t}\right)+\alpha(A f(u), u)-\alpha(g, u)=0 .
\end{aligned}
$$

Observe now that

$$
\left(A f(u), u_{t}\right)=\frac{1}{2} \frac{\mathrm{d}}{\mathrm{d} t}\left(f^{\prime}(u) \nabla u, \nabla u\right)-\frac{1}{2}\left(f^{\prime \prime}(u)|\nabla u|^{2}, u_{t}\right) .
$$

Thus, (3.15) takes the form

$$
\begin{aligned}
\frac{\mathrm{d}}{\mathrm{d} t} & {\left[\frac{\varepsilon}{2}\left\|u_{t}\right\|^{2}+\frac{1}{2}\|A u\|^{2}+\frac{1}{2}\left(f^{\prime}(u) \nabla u, \nabla u\right)+\varepsilon \alpha\left(u_{t}, u\right)-(g, u)\right] } \\
& +(1-\varepsilon \alpha)\left\|u_{t}\right\|^{2}+\alpha\|A u\|^{2}+\alpha\left(f^{\prime}(u) \nabla u, \nabla u\right)+\alpha\left(u_{t}, u\right)-\alpha(g, u) \\
= & \frac{1}{2}\left(f^{\prime \prime}(u)|\nabla u|^{2}, u_{t}\right) .
\end{aligned}
$$

Using standard interpolation arguments, the right hand side can be estimated as follows

$$
\frac{1}{2}\left(f^{\prime \prime}(u)|\nabla u|^{2}, u_{t}\right) \leq \frac{1}{4}\left\|u_{t}\right\|^{2}+\frac{1}{4}\left\|f^{\prime \prime}(u)\right\|_{L^{\infty}(\Omega)}^{2}\|\nabla u\|_{L^{4}(\Omega)}^{4} .
$$


We then introduce the following functional

$$
\mathcal{Z}(t):=\frac{\varepsilon}{2}\left\|u_{t}\right\|^{2}+\frac{1}{2}\|u\|_{D(A)}^{2}+\frac{1}{2}\left(f^{\prime}(u) \nabla u, \nabla u\right)+\varepsilon \alpha\left(u_{t}, u\right)-(g, u),
$$

which is bounded from below. Actually, thanks to (2.8) and the Young inequality we have (possibly choosing $\alpha$ small)

$$
\mathcal{Z}(t) \geq c\left(\varepsilon\left\|u_{t}\right\|^{2}+\|u\|_{D(A)}^{2}\right)-\frac{\lambda}{2}\|\nabla u\|^{2}-\|u\|^{2}-\|g\|^{2} .
$$

Thus, thanks to (3.8), we infer that

$$
\mathcal{Z}(t) \geq c\left(\varepsilon\left\|u_{t}\right\|^{2}+\|u\|_{D(A)}^{2}\right)-Q_{0}\left(\left\|U_{0}\right\|_{1}^{2}\right)-\|g\|^{2} .
$$

Moreover, by (2.20), $f \in C^{2}(\mathbb{R})$, and the embedding $H^{2}(\Omega) \hookrightarrow C^{0}(\bar{\Omega})$, we have

$$
\mathcal{Z}(0) \leq Q_{1}\left(\left\|U_{0}\right\|_{1}\right)+Q_{1}(\|g\|) .
$$

Thus, on account of (3.18), from (3.17) we deduce the inequality

$$
\frac{\mathrm{d}}{\mathrm{d} t} \mathcal{Z}+\kappa \mathcal{Z} \leq Q_{2}(\mathcal{Z})
$$

for some $\kappa$ depending on the choice of $\alpha$. Let now $y$ be the solution to the Cauchy problem

$$
y^{\prime}(t)+\kappa y(t)=Q_{2}(y(t)), \quad y(0)=\mathcal{Z}(0) .
$$

Then, the comparison principle gives

$$
\mathcal{Z}(t) \leq y(t)
$$

for every $t \in\left[0, T_{0}\right]$, where $T_{0}=T_{0}\left(\left\|\left(u_{0}, u_{1}\right)\right\|_{1},\|g\|_{V^{\prime}}\right)$ is some positive time which is (strictly) smaller than both the life span of $y$ and of the Galerkin solution (i.e., $T^{*}$ ). Thus, recalling (3.21), we find

$$
c\|U(t)\|_{1}^{2}-Q_{0}\left(\left\|U_{0}\right\|_{1}^{2}\right)-\|g\|^{2} \leq \mathcal{Z}(t) \leq \mathcal{Q}\left(\left\|U_{0}\right\|_{1},\|g\|\right)
$$

for all $t \in\left[0, T_{0}\right]$, with $\mathcal{Q}$ as in the statement.

In order to pass to the limit as $N$ goes to $+\infty$, we make explicit the $N$-dependence again. Recalling (3.4)-(3.6), estimate (3.26) gives

$$
\left\|\left(u^{N}, u_{t}^{N}\right)\right\|_{L^{\infty}\left(0, T_{0} ; \mathcal{V}_{1}\right)} \leq C
$$

for a positive constant $C$ independent of $N$ but, of course, depending on $U_{0}$. Moreover, after choosing $v=A^{-1} u_{t t}^{N}$ in (3.2), we get

$$
\left\|u_{t t}^{N}\right\|_{L^{\infty}\left(0, T_{0} ; D\left(A^{-1}\right)\right)} \leq C .
$$


These estimates are enough to pass to the limit as $N$ tends to $+\infty$ in (3.2) and to obtain a solution to (2.14). Actually, (3.27) and (3.28) combined with the embedding $H^{2}(\Omega) \hookrightarrow C^{0}(\bar{\Omega})$ and with the smoothness of $f$, give that (up to a subsequence)

$$
\begin{aligned}
& \left(u^{N}, u_{t}^{N}\right) \rightarrow U \text { weakly star in } L^{\infty}\left(0, T_{0} ; \mathcal{V}_{1}\right), \\
& u_{t t}^{N} \rightarrow u_{t t} \text { weakly star in } L^{\infty}\left(0, T_{0} ; D\left(A^{-1}\right)\right), \\
& f\left(u^{N}\right) \rightarrow f(u) \text { uniformly in } \bar{\Omega} \times\left[0, T_{0}\right],
\end{aligned}
$$

as $N \rightarrow+\infty$. Thus, by lower semicontinuity of norms with respect to weak star convergence, $u$ is a weak solution to problem $P_{\varepsilon}$ satisfying (2.21). This concludes the proof.

REMARK 3.1. Once we have the uniform boundedness of $f(u)$ at our disposal, it is easy to see that, if $f$ and the initial datum are smooth enough, then more regular solutions can be constructed. For instance, if $U_{0} \in \mathcal{V}_{2}$ and (2.7) and (2.8) hold, then a local in time quasi-strong solution exists. The details of this standard argument are left to the reader.

REMARK 3.2. Let us notice that, for $U$ as in Theorem 2.5, one has, more precisely,

$$
U \in C^{0}\left(\left[0, T_{0}\right] ; \mathcal{V}_{1}\right)
$$

To show this, we can rewrite Eq. (2.14) as

$$
A^{-1}\left(\varepsilon u_{t t}+u_{t}\right)+A u=A^{-1} g-f(u)=: h,
$$

where it is clear, due to (2.21), that $h \in L^{2}\left(0, T_{0} ; D(A)\right)$. Thus, property (3.32) can be shown simply by approximating both $h$ and the initial datum $U_{0}$ by smoother sequences $\left\{h_{n}\right\}$ and $\left\{U_{0, n}\right\}$, applying the linear theory to the resulting problem

$$
A^{-1}\left(\varepsilon u_{n, t t}+u_{n, t}\right)+A u_{n}=h_{n},
$$

and then using a Cauchy sequence argument. Note that one has to require that $h_{n} \rightarrow h$ strongly in $L^{2}\left(0, T_{0} ; D(A)\right)$. Further details on this procedure can be found in [20, Proof of Thm. 3.1].

\subsection{Proof of Theorem 2.6}

Referring to the notation in the statement, we set $u=u^{1}-u^{2}$ and $U=\left(u, u_{t}\right)$, so that

$$
A^{-1}\left(\varepsilon u_{t t}+u_{t}\right)+A u+f\left(u_{1}\right)-f\left(u_{2}\right)=0, \quad \text { in } H, \text { a.e. in }(0, T) .
$$

We now test (3.35) by $u_{t}$, obtaining

$$
\frac{1}{2} \frac{\mathrm{d}}{\mathrm{d} t}\|U\|_{0}^{2}+\left\|u_{t}\right\|_{V^{\prime}}^{2}=-\left(A^{1 / 2}\left(f\left(u^{1}\right)-f\left(u^{2}\right)\right), A^{-1 / 2} u_{t}\right) .
$$


Observe now that

$$
\begin{aligned}
\| & A^{1 / 2}\left(f\left(u^{1}\right)-f\left(u^{2}\right)\right) \|^{2} \\
= & \left\|\nabla\left(\int_{0}^{1} f^{\prime}\left(s u_{1}+(1-s) u_{2}\right) \mathrm{d} s \cdot\left(u_{1}-u_{2}\right)\right)\right\|^{2} \\
\leq & \sup _{s \in[0,1]}\left\|f^{\prime}\left(s u_{1}+(1-s) u_{2}\right)\right\|_{L^{\infty}(\Omega)}^{2}\left\|A^{1 / 2} u\right\|^{2} \\
& +\sup _{s \in[0,1]}\left\|f^{\prime \prime}\left(s u_{1}+(1-s) u_{2}\right)\right\|_{L^{\infty}(\Omega)}^{2}\left(1+\left\|u_{1}\right\|_{D(A)}^{2}+\left\|u_{2}\right\|_{D(A)}^{2}\right)\left\|A^{1 / 2} u\right\|^{2},
\end{aligned}
$$

thanks to the regularity properties of $f$. Thus, for a proper constant $C$, we find out

$$
\left(A^{1 / 2}\left(f\left(u^{1}\right)-f\left(u^{2}\right)\right), A^{-1 / 2} u_{t}\right) \leq C\|\nabla u\|^{2}+\frac{1}{2}\left\|u_{t}\right\|_{V^{\prime}}^{2},
$$

whence it follows that

$$
\frac{1}{2} \frac{\mathrm{d}}{\mathrm{d} t}\|U\|_{0}^{2}+\frac{1}{2}\left\|u_{t}\right\|_{V^{\prime}}^{2} \leq C\|\nabla u\|^{2},
$$

and the assertion immediately follows from the Gronwall lemma.

\subsection{Proof of Theorem 2.7}

We first need a couple of important preparatory propositions. The first one is devoted to prove some uniform in time regularity estimates for the solution $u^{0}$ to an auxiliary (parabolic) problem (cf. Problem $P_{0}$ below) which contains, as a forcing term, a solution $u$ to $P_{\varepsilon}$ with initial datum $U_{0}=\left(u_{0}, u_{1}\right) \in \mathcal{V}_{1}$. As a second step we will prove that $\left\|\left(u-u^{0}\right)\right\|_{V^{\prime}}$ can be controlled by $c \varepsilon^{1 / 2}$, for some $c>0$, on bounded time intervals. The coupling of these two properties will allow us to reconsider and improve the bound (2.21). In fact, we will see that, if $\varepsilon$ is small enough and, correspondingly, the $\mathcal{V}_{1}$-norm of $\left(u_{0}, u_{1}\right)$ is controlled in the sense of (2.23), then the left hand side of (2.21) is bounded uniformly in time in a quantitatively controlled way (in particular, it cannot explode as $t \nearrow T_{0}$ ). By standard extension arguments, this implies that the $\mathcal{V}_{1}$-norm of $U=\left(u, u_{t}\right)$ is uniformly bounded in $(0, \infty)$, i.e., $u$ is a global weak solution (and, consequently, it is unique in this class). Actually, we will prove something more, namely, under the above restrictions, (2.21) can be improven to get a global and dissipative inequality which entails the existence of a bounded absorbing set.

Let us then state the auxiliary Problem $P_{0}$ as

$$
\begin{aligned}
& A^{-1} u_{t}^{0}+A u^{0}+f\left(u^{0}\right)+\lambda u^{0}=\lambda u+A^{-1} g, \quad \text { in } V^{\prime}, \text { a.e. in }(0, T), \\
& \left.u^{0}\right|_{t=0}=u_{0} \quad \text { in } \Omega .
\end{aligned}
$$

Here, the initial datum $u_{0}$ in (3.41) is the first component of the initial data of Problem $P_{\varepsilon}$. Thus it lies in $D(A)$. Moreover, it is worth noting that the function $u$ in the right 
hand side is the (unique) weak local solution to Problem $P_{\varepsilon}$ constructed in Theorem 2.5 and defined in some interval $\left(0, T_{0}\right)$. Thus, our first aim will be to construct the solution $u^{0}$ in the same time interval. Observe also that, by (2.8), $\beta:=f+\lambda$ Id is a nondecreasing monotone function. Arguing as in the proof of Theorem 2.5, i.e., testing (2.14) by $A\left(u_{t}+\alpha u\right)$, we have that $U$ satisfies the analogue of (2.25), namely

$$
\|U(t)\|_{0}^{2}+\int_{t}^{t+1}\left\|u_{t}(s)\right\|_{V^{\prime}}^{2} \mathrm{~d} s \leq Q\left(\left\|U_{0}\right\|_{1}\right) e^{-\kappa t}+Q(\|g\|), \quad \forall t \in\left(0, T_{0}\right),
$$

for some constant $\kappa$ independent of $\varepsilon$.

Let us now state our first proposition addressing the properties of the solution $u^{0}$ to $P_{0}$. This is in fact a variant of Theorem 2.9 (cf. also [8]) which makes the regularity and dissipativity properties of $u^{0}$ more precise.

PROPOSITION 3.3. Let the assumptions (2.7), (2.8) and (2.10) hold. Let $u^{0}$ be a solution to Problem $P_{0}$. Then, the following estimate holds

$$
\left\|u^{0}(t)\right\|_{D(A)} \leq Q\left(\left\|U_{0}\right\|_{1}\right) e^{-\kappa t}+Q(\|g\|), \quad \forall t \in\left(0, T_{0}\right),
$$

for some positive constant $\kappa$ independent of $\varepsilon$ and some monotone function $Q$ independent of $T_{0}$.

Proof. The proof is based on a number of a priori estimates which will be obtained by formal arguments. However, the whole procedure could be justified within a new Faedo-Galerkin approximation scheme, which is completely standard and hence omitted.

The main point in the proof is that we will use only property (3.14) of the function $u$, where the right hand side does not explode as $t \nearrow T_{0}$. Thus, let us test (3.40) by $u_{t}^{0}+\alpha u^{0}$. It is then not difficult to infer, at least for $\alpha>0$ small enough,

$$
\frac{\mathrm{d}}{\mathrm{d} t} E_{*}+\alpha E_{*}+\left\|u_{t}^{0}\right\|_{V^{\prime}}^{2} \leq \lambda\left(u_{t}^{0}+\alpha u^{0}, u\right)
$$

where we have set

$$
E_{*}=E_{*}\left(u^{0}\right):=\frac{1}{2}\left\|\nabla u^{0}\right\|^{2}+\frac{\alpha}{2}\left\|u^{0}\right\|_{V^{\prime}}^{2}+\left(B\left(u^{0}\right), 1\right)-\left\langle g, A^{-1} u^{0}\right\rangle,
$$

with $B(r):=F(r)+\lambda r^{2} / 2$. Thus, estimating standardly the right hand side of (3.44), we obtain the inequality

$$
\frac{\mathrm{d}}{\mathrm{d} t} E_{*}+\frac{\alpha}{2} E_{*}+\frac{1}{2}\left\|u_{t}^{0}\right\|_{V^{\prime}}^{2} \leq c\|u\|_{V}^{2}
$$

where $c$ does not depend on $\varepsilon$. Thus, multiplying (3.46) by $e^{\frac{\alpha}{2} t}$ and then integrating between 0 and $t$, it is not difficult to obtain that $u^{0}$ fulfils the dissipative bound

$$
\begin{aligned}
& E_{*}\left(u^{0}(t)\right)+\frac{1}{2} \int_{0}^{t} \mathrm{e}^{-\frac{\alpha}{2}(t-s)}\left\|u_{t}^{0}(s)\right\|_{V^{\prime}}^{2} \mathrm{~d} s \\
& \leq E_{*}\left(u_{0}\right) \mathrm{e}^{-\frac{\alpha}{2} t}+c \int_{0}^{t} \mathrm{e}^{-\frac{\alpha}{2}(t-s)}\|u(s)\|_{V}^{2} \mathrm{~d} s .
\end{aligned}
$$


Recalling (3.14), and choosing $\alpha \geq 2 \kappa$, with $\kappa$ as in (2.25), one then infers

$$
E_{*}\left(u^{0}(t)\right)+\frac{1}{2} \int_{0}^{t} \mathrm{e}^{-\frac{\alpha}{2}(t-s)}\left\|u_{t}^{0}(s)\right\|_{V^{\prime}}^{2} \mathrm{~d} s \leq Q\left(\left\|U_{0}\right\|_{1}\right) \mathrm{e}^{-\frac{\alpha}{2} t}+Q(\|g\|) .
$$

Next, let us test (3.40) by $A^{3} u^{0}$. We obtain (recall that $\beta=f+\lambda$ Id)

$$
\frac{\mathrm{d}}{\mathrm{d} t}\left\|A u^{0}\right\|^{2}+\left\|A^{2} u^{0}\right\|^{2} \leq c\left(\|g\|^{2}+\left\|A \beta\left(u^{0}\right)\right\|^{2}+\|A u\|^{2}\right) .
$$

Using interpolation, we then obtain

$$
\frac{\mathrm{d}}{\mathrm{d} t}\left\|A u^{0}\right\|^{2}+\left\|A^{2} u^{0}\right\|^{2} \leq c\left(\|g\|^{2}+Q\left(\left\|A u^{0}\right\|\right)+\|A u\|^{2}\right),
$$

whence, recalling (2.21) and applying again the comparison argument as in (3.24) and (3.25), it follows that there exists a time $T_{1} \leq T_{0}$, depending on $\left\|U_{0}\right\|_{1}$ and on $\|g\|$, such that

$$
\left\|A u^{0}(t)\right\|^{2} \leq q(\|g\|)+q\left(\left\|U_{0}\right\|_{1}\right), \quad \forall t \in\left[0, T_{1}\right] .
$$

We now test (3.40) by $u_{t}^{0}$. This yields

$$
\frac{1}{2}\left\|u_{t}^{0}\right\|_{V^{\prime}}^{2}+\frac{\mathrm{d}}{\mathrm{d} t}\left(\frac{1}{2}\left\|\nabla u^{0}\right\|^{2}+\left(B\left(u^{0}\right), 1\right)\right) \leq c\left(\|u\|_{V}^{2}+\|g\|^{2}\right) .
$$

Integrating over $\left(0, T_{1}\right)$, we then deduce (recall (3.8))

$$
\int_{0}^{T_{1}}\left\|u_{t}^{0}(t)\right\|_{V^{\prime}}^{2} \mathrm{~d} t \leq c T_{1}\left(Q\left(\left\|U_{0}\right\|_{1}\right)+Q(\|g\|)\right) .
$$

We now differentiate with respect to time (3.40) and test the resulting equation by $t u_{t}^{0}$, obtaining

$$
\frac{\mathrm{d}}{\mathrm{d} t}\left(t\left\|u_{t}^{0}\right\|_{V^{\prime}}^{2}\right)+t\left\|\nabla u_{t}^{0}\right\|^{2} \leq\left\|u_{t}^{0}\right\|_{V^{\prime}}^{2}+c t\left\|u_{t}\right\|_{V^{\prime}}^{2} .
$$

Integrating (3.54) over ( $\left.0, T_{1}\right)$, recalling (3.53), and using the dissipation integral (3.9), we obtain

$$
\left\|u_{t}^{0}\left(T_{1}\right)\right\|_{V^{\prime}}^{2} \leq Q\left(\left\|U_{0}\right\|_{1}\right)+Q(\|g\|) .
$$

Let us differentiate (3.40) once more and test the obtained equation by $u_{t}^{0}$. We then get

$$
\frac{\mathrm{d}}{\mathrm{d} t}\left\|u_{t}^{0}\right\|_{V^{\prime}}^{2}+\left\|\nabla u_{t}^{0}\right\|^{2} \leq c\left\|u_{t}\right\|_{V^{\prime}}^{2} .
$$

Summing (3.46) and (3.56), we infer

$$
\frac{\mathrm{d}}{\mathrm{d} t} \mathcal{F}(t)+\kappa \mathcal{F}(t) \leq c\left(\|u\|_{V}^{2}+\left\|u_{t}\right\|_{V^{\prime}}^{2}\right),
$$


for some positive constants $\kappa$ (possibly different from the $\kappa$ in (3.42)) and $c$. Here (see $(3.45))$

$$
\mathcal{F}:=E_{*}\left(u^{0}\right)+\left\|u_{t}^{0}\right\|_{V^{\prime}}^{2}
$$

Subsequently, we integrate (3.57) over $\left(T_{1}, t\right)$, with $t$ arbitrary, thus obtaining

$$
\mathcal{F}(t) \leq c \mathrm{e}^{-\kappa\left(t-T_{1}\right)} \mathcal{F}\left(T_{1}\right)+c \int_{T_{1}}^{t} \mathrm{e}^{-\kappa(t-s)}\left(\|u(s)\|_{V}^{2}+\left\|u_{t}(s)\right\|_{V^{\prime}}^{2}\right) \mathrm{d} s .
$$

According to (3.42), we have that

$$
\int_{t}^{t+1}\left(\|u(s)\|_{V}^{2}+\left\|u_{t}(s)\right\|_{V^{\prime}}^{2}\right) \mathrm{d} s \leq Q\left(\left\|U_{0}\right\|_{1}\right) \mathrm{e}^{-\kappa t}+Q(\|g\|) .
$$

Thus, using a suitable version of the Gronwall lemma (see, e.g., [29]) and recalling (3.55), we get

$$
\left\|u_{t}^{0}(t)\right\|_{V^{\prime}}^{2} \leq Q\left(\left\|U_{0}\right\|_{1}\right) \mathrm{e}^{-\kappa t}+Q(\|g\|), \quad \forall t \geq T_{1} .
$$

for some $\kappa>0$ (the smaller between the one appearing in (3.42) and the one in (3.57)). Next, by a direct comparison argument in (3.40) and (3.42) again, and standard elliptic regularity, we infer

$$
\left\|u^{0}(t)\right\|_{D(A)} \leq Q\left(\left\|U_{0}\right\|_{1}\right) \mathrm{e}^{-\kappa t}+Q(\|g\|), \quad \forall t \geq T_{1} .
$$

Finally, (3.51) and (3.61) imply (3.43), which concludes the proof.

The following proposition gives the quantitative estimate of the difference between $u^{0}$ and the (unique) local solution $u$ to $P_{\varepsilon}$.

PROPOSITION 3.4. Let assumptions (2.7), (2.8) and (2.10) hold, let $u$ as before, and let $u^{0}(t)$ be the corresponding (unique) solution to Problem $P_{0}$. Then, the following estimate holds

$$
\left\|u^{0}(t)-u(t)\right\|_{V^{\prime}}^{2} \leq c \varepsilon\left(Q\left(\left\|u_{0}, u_{1}\right\|_{1}\right) \mathrm{e}^{-\kappa t}+Q(\|g\|)\right), \quad \forall t \in\left(0, T_{0}\right),
$$

where $Q, c, \kappa$ are independent both of $\varepsilon$ and of $T_{0}$.

Proof. Let us take the difference between (2.14) and (3.40). Setting $\bar{u}:=u-u^{0}$, we have that

$$
A^{-1} \bar{u}_{t}+A \bar{u}+\beta(u)-\beta\left(u^{0}\right)=-A^{-1}\left(\varepsilon u_{t t}\right), \quad \text { a.e. in }\left(0, T_{0}\right) .
$$

We can then test (3.63) by $\bar{u}$. A number of simple manipulations and the monotonicity of $\beta$ allow us to deduce the following inequality

$$
\frac{\mathrm{d}}{\mathrm{d} t}\left[\frac{1}{2}\|\bar{u}\|_{V^{\prime}}^{2}+\varepsilon\left\langle u_{t}, A^{-1} \bar{u}\right\rangle\right]+\|\bar{u}\|_{V}^{2}=\varepsilon\left\langle u_{t}, A^{-1} \bar{u}_{t}\right\rangle \leq \frac{\varepsilon}{2}\left(\left\|u_{t}\right\|_{V^{\prime}}^{2}+\left\|\bar{u}_{t}\right\|_{V^{\prime}}^{2}\right) .
$$


Adding to (3.64) the straightforward inequality

$$
\varepsilon\left\langle u_{t}, A^{-1} \bar{u}\right\rangle \leq c \varepsilon\left\|u_{t}\right\|_{V^{\prime}}^{2}+\frac{1}{2}\|\bar{u}\|_{V}^{2},
$$

and noting by $Y$ the quantity in square brackets in (3.64), we easily obtain

$$
\frac{\mathrm{d}}{\mathrm{d} t} Y+\kappa Y \leq c \varepsilon\left(\left\|u_{t}\right\|_{V^{\prime}}^{2}+\left\|u_{t}^{0}\right\|_{V^{\prime}}^{2}\right)=: m(t) .
$$

Observe now that (see (3.42), (3.53) and (3.60))

$$
\int_{t}^{t+1} m(s) \mathrm{d} s \leq \varepsilon\left(Q\left(\left\|U_{0}\right\|_{1}\right) \mathrm{e}^{-\kappa t}+Q(\|g\|)\right),
$$

and recall that $Y(0)=0$. Then, the comparison principle entails that

$$
Y(t) \leq \varepsilon\left(Q\left(\left\|U_{0}\right\|_{1}\right) \mathrm{e}^{-\kappa t}+Q(\|g\|)\right),
$$

which yields, using (3.42) once again,

$$
\|\bar{u}(t)\|_{V^{\prime}}^{2} \leq \varepsilon\left(Q\left(\left\|U_{0}\right\|_{1}\right) \mathrm{e}^{-\kappa t}+Q(\|g\|)\right),
$$

that is, the desired estimate.

Assuming that $\varepsilon$ is sufficiently small and the $\mathcal{V}_{1}$-norm of the initial data is controlled by a function $R(\varepsilon)$ with the properties indicated in the statement of Theorem 2.7, let us now derive a $\mathcal{V}_{1}$-bound for $U$ which is independent of the final time $T_{0}$. This will permit, by standard extension methods, to prolong $U$ to a global in time weak solution. The key point to show such an estimate is the comparison of $U$ with the auxiliary solution $u^{0}$ provided by Proposition 3.4.

The starting point is the following relation (cf. (3.17)-(3.19)), holding for all $t \in\left(0, T_{0}\right)$,

$$
\frac{\mathrm{d}}{\mathrm{d} t} \mathcal{Z}(t)+\kappa \mathcal{Z}(t) \leq \frac{1}{4}\left\|f^{\prime \prime}(u)\right\|_{L^{\infty}(\Omega)}^{2}\|u\|_{W^{1,4}(\Omega)}^{4} \leq M\left(\|u\|_{H^{7 / 4}(\Omega)}\right) .
$$

Here the last inequality is a consequence of the continuous embedding $H^{7 / 4}(\Omega) \subset$ $L^{\infty}(\Omega) \cap W^{1,4}(\Omega)$ and of the $C^{2}$ regularity of $f$. We have denoted by $M$ a continuous and increasingly monotone function independent of $T_{0}$ and such that $M(0)=0$. Recalling (3.43) and (3.69), and properly using interpolation, we can write

$$
\begin{aligned}
& \|u\|_{H^{7 / 4}(\Omega)} \leq\|\bar{u}\|_{H^{7 / 4}(\Omega)}+\left\|u^{0}\right\|_{H^{7 / 4}(\Omega)} \\
& \quad \leq\|\bar{u}\|_{D(A)}^{11 / 12}\|\bar{u}\|_{V^{\prime}}^{1 / 12}+\left\|u^{0}\right\|_{H^{7 / 4}(\Omega)} \\
& \quad \leq\|\bar{u}\|_{D(A)}^{11 / 12}\left[\varepsilon^{1 / 24}\left(Q\left(\left\|U_{0}\right\|_{1}\right) \mathrm{e}^{-\kappa t}+Q(\|g\|)\right)\right]+Q\left(\left\|U_{0}\right\|_{1}\right) \mathrm{e}^{-\kappa t}+Q(\|g\|) \\
& \quad \leq\left(\|u\|_{D(A)}^{11 / 12}+\left\|u^{0}\right\|_{D(A)}^{11 / 12}\right)\left[\varepsilon^{1 / 24}\left(Q\left(\left\|U_{0}\right\|_{1}\right) \mathrm{e}^{-\kappa t}+Q(\|g\|)\right)\right] \\
& \quad+Q\left(\left\|U_{0}\right\|_{1}\right) \mathrm{e}^{-\kappa t}+Q(\|g\|) .
\end{aligned}
$$


Now, recalling (3.8) and (3.21), we can define

$$
\tilde{\mathcal{Z}}=\mathcal{Z}+Q\left(\left\|U_{0}\right\|_{1}\right) \mathrm{e}^{-\kappa_{0} t}+Q\left(\|g\|_{V^{\prime}}\right),
$$

for suitable $Q$ and $\kappa_{0}>0$, chosen such that

$$
c\|U(t)\|_{1}^{2} \leq \tilde{\mathcal{Z}}(t), \quad \forall t \in\left(0, T_{0}\right),
$$

for some $c>0$. Using (3.43) and (3.73), we then see that the $M$-term on the right hand side of (3.70) can be estimated as follows:

$$
M\left(\|u\|_{H^{7 / 4}(\Omega)}\right) \leq M\left[\left(\varepsilon^{1 / 24} \tilde{\mathcal{Z}}+1\right)\left(Q\left(\left\|U_{0}\right\|_{1}\right) \mathrm{e}^{-\kappa t}+Q(\|g\|)\right)\right]
$$

(we assumed here $\tilde{\mathcal{Z}} \geq 1$, which is of course not restrictive). Thus, (3.70) can be rewritten as

$$
\frac{\mathrm{d}}{\mathrm{d} t} \tilde{\mathcal{Z}}(t)+\kappa \tilde{\mathcal{Z}}(t) \leq M_{1}\left(\boldsymbol{P} \varepsilon^{1 / 24} \tilde{\mathcal{Z}}\right)+M_{2}(\boldsymbol{Q}), \quad \forall t \in\left(0, T_{0}\right),
$$

where $M_{1}$ and $M_{2}$ have the same properties as $M$ (i.e., they are continuous monotone functions such that $M_{1}(0)=M_{2}(0)=0$ ) and we have set, for brevity,

$$
\boldsymbol{P}:=Q_{3}\left(\left\|U_{0}\right\|_{1}\right) \mathrm{e}^{-\kappa t}+Q_{4}(\|g\|), \quad \boldsymbol{Q}:=Q_{5}\left(\left\|U_{0}\right\|_{1}\right) \mathrm{e}^{-\kappa t}+Q_{6}(\|g\|),
$$

for some $Q_{i}, i=3, \ldots, 6$, having the same properties as the generic $Q$.

We can now set $\rho=\tilde{\mathcal{Z}}(0)>0$ and observe that (cf. (3.19), (3.22) and (3.72)), for a fixed $R>0$, we have that

$$
\left\|U_{0}\right\|_{1} \leq R \Rightarrow \rho \leq Q_{7}(R)+Q_{8}(\|g\|) .
$$

Now, for some $z^{*}>\rho$ whose value will be chosen later, we put

$$
\varepsilon^{1 / 24}:=\frac{1}{\left(Q_{3}(R)+Q_{4}(\|g\|)\right) z^{*}} M_{1}^{-1}(1) .
$$

Notice that here we used that $M_{1}$ is continuous and such that $M_{1}(0)=0$. Hence, if $\varepsilon>0$ is as above (or smaller), and $z \leq z^{*}$, then there holds

$$
M_{1}\left[\left(Q_{3}(R)+Q_{4}(\|g\|)\right) z \varepsilon^{1 / 24}\right] \leq M_{1}\left[\left(Q_{3}(R)+Q_{4}(\|g\|)\right) z^{*} \varepsilon^{1 / 24}\right] \leq 1 .
$$

Thus, assuming that $\tilde{\mathcal{Z}}$ is smaller than $z^{*},(3.75)$ entails in particular

$$
\frac{\mathrm{d}}{\mathrm{d} t} \tilde{\mathcal{Z}}(t)+\kappa \tilde{\mathcal{Z}}(t) \leq M_{3}(\boldsymbol{Q}):=1+M_{2}(\boldsymbol{Q}) .
$$

By the comparison principle, we then have

$$
\begin{aligned}
\tilde{\mathcal{Z}}(t) & \leq \rho \mathrm{e}^{-\kappa t}+\int_{0}^{t} \mathrm{e}^{-\kappa(t-s)} M_{3}\left(Q_{5}(R) \mathrm{e}^{-\kappa s}+Q_{6}(\|g\|)\right) \mathrm{d} s \\
& \leq \rho+\kappa^{-1} M_{3}\left(Q_{5}(R)+Q_{6}(\|g\|)\right) .
\end{aligned}
$$


The above relation holds, in principle, until $\tilde{\mathcal{Z}}(t)$ is not greater than the number $z^{*}$. To make this true uniformly in time, it is then enough to set

$$
z^{*}:=\rho+\kappa^{-1} M_{3}\left(Q_{5}(R)+Q_{6}(\|g\|)\right),
$$

and take $\varepsilon$ as given by (3.78) with this choice of $z^{*}$. More precisely, from (3.78) we obtain

$$
\varepsilon=\left(\frac{1}{\left(Q_{3}(R)+Q_{4}(\|g\|)\right)\left(\rho+M_{3}\left(Q_{5}(R)+Q_{6}(\|g\|)\right)\right)} M_{1}^{-1}(1)\right)^{24}=: \Phi(R),
$$

where $\Phi$ is a monotone function of $R$ as we think $\rho$ be controlled by the latter expression in (3.77). The desired $R$ is then chosen simply as $\Phi^{-1}$. Of course, the value $\varepsilon_{0}$ is then given by $\lim _{R \searrow 0} \Phi(R)$.

Bound (3.81) entails that $\tilde{\mathcal{Z}}(t)$ is bounded in a way that does not depend on $T_{0}$. Thus, the $\mathcal{V}_{1}$-norm of $U(t)$ cannot explode in finite times, which permits to extend $U$ to a global solution (unique in its class by Theorem 2.6), as desired. Moreover (3.81) can now be improved. Namely, we have

$$
\begin{aligned}
\tilde{\mathcal{Z}}(t) & \leq \rho \mathrm{e}^{-\kappa t}+\mathrm{e}^{-\kappa t}\left[\int_{0}^{T} \mathrm{e}^{\kappa s} M_{3}\left(Q_{5}(R)+Q_{6}(\|g\|)\right) \mathrm{d} s+\int_{T}^{t} \mathrm{e}^{\kappa s} Q(\|g\|) \mathrm{d} s\right] \\
& \leq Q(R) \mathrm{e}^{-\kappa t}+Q(\|g\|),
\end{aligned}
$$

where $\boldsymbol{T}$ is a suitable time depending on $\rho$ (and, consequently, on $R$ ) and on $M_{3}$ such that, for $t \geq \boldsymbol{T}, Q_{5}(R) \mathrm{e}^{-\kappa s}$ is smaller than some computable quantity $Q(\|g\|)$ (notice that the $\kappa$ 's in (3.81) and (3.84) might well differ from each other). Relation (3.84) has then the form of a dissipative estimate and entails (2.24).

To conclude it only remains to prove the energy identity (2.26), which now follows with few complications from the results above. Formally speaking, we have to test (3.33) by $u_{t}+\frac{1}{2 \varepsilon} u$. Setting then (for $U=\left(u, u_{t}\right)$, as usual)

$$
I(U):=\frac{\varepsilon}{2}\left\|u_{t}\right\|^{2}+\frac{1}{2}\|A u\|^{2}+\frac{1}{2}\left(u_{t}, u\right)+\frac{1}{2}\left(f^{\prime}(u)|\nabla u|^{2}, 1\right),
$$

we deduce that $I$ satisfies

$$
\frac{\mathrm{d}}{\mathrm{d} t} I(U)+\frac{1}{\varepsilon} I(U)=\left(g, u_{t}+\frac{1}{2 \varepsilon} u\right)+\frac{1}{2}\left(f^{\prime \prime}(u)|\nabla u|^{2}, u_{t}\right),
$$

so that (2.26) follows simply by integrating (3.86) over a time interval $(0, M)$.

However, the regularity of $u_{t}$ is not enough to justify this estimate (while that of $u$ suffices). Thus, to make the procedure rigorous, we can rewrite once more (2.14) as

$$
\varepsilon u_{t t}+u_{t}+A^{2} u=g+f^{\prime}(u) \Delta u+f^{\prime \prime}(u)|\nabla u|^{2}=: k,
$$

and observe that, once $U$ is globally bounded with values in $\mathcal{V}_{1}$, proceeding as in Remark 3.2 one can prove that $U \in C^{0}\left([0, T] ; \mathcal{V}_{1}\right)$ for all $T>0$. 
At this point, we claim that, for all $0 \leq s \leq t \leq T$, there holds

$$
\begin{aligned}
& \int_{s}^{t}\left(f^{\prime}(u) \Delta u+f^{\prime \prime}(u)|\nabla u|^{2}, u_{t}\right) \\
& \quad=-\frac{1}{2}\left(f^{\prime}(u(t))|\nabla u(t)|^{2}-f^{\prime}(u(s))|\nabla u(s)|^{2}, 1\right)+\int_{s}^{t}\left(f^{\prime \prime}(u)|\nabla u|^{2}, u_{t}\right) .
\end{aligned}
$$

Indeed, this can be proven by substituting $U$ with some smoother $U_{n}$ (for which the above equality is trivial) such that, as $n$ goes to $+\infty, U_{n} \rightarrow U$ strongly in $C^{0}([0, T]$; $\mathcal{V}_{1}$ ) and taking the limit. Actually, it is easy to see that all terms in both hands sides of (3.88) converge to the expected values (cf. [20, Sec. 3] for more details on a similar argument). Finally, to justify the fact that the product of the left hand side of (3.87) times $u_{t}$ gives the expected result one can see (3.87) as a linear problem with source datum $k$, regularize $k$ with a sequence $\left\{k_{n}\right\}$, and take once more the limit by a (standard) Cauchy argument. The proof is complete.

\subsection{Asymptotic boundedness and dissipativity in $\mathcal{V}_{2}$}

We give here the first part of the proof of our main Theorem 2.10, whose hypotheses are implicitly assumed for the whole of this section (notice, however, that for the proof of this part the assumption $f \in C_{\text {loc }}^{2,1}$ is not needed). In particular, we will show that, under the assumptions of Theorem 2.7 (in particular the initial data must satisfy (2.23)), the semigroup $S_{\varepsilon}$ admits an exponentially attracting set $\mathcal{A}_{2}$ bounded in $\mathcal{V}_{2}$. This fact ensures that there exists a global attractor bounded in $\mathcal{V}_{2}$. Note that we must appeal to the results in [30] since the (Lipschitz) continuity of $S_{\varepsilon}$ is only guaranteed with respect to the $\mathcal{V}_{0}$ norm (see (3.39)).

To prove this fact, by (2.24) we can consider weak solutions $U$ whose initial datum $U_{0}$ lies in an absorbing set $\mathcal{B}_{1}$ bounded in $\mathcal{V}_{1}$. Moreover, we can assume $\mathcal{B}_{1}$ be positively invariant for $S_{\varepsilon}$. Thus, in particular, we have that

$$
\|U(t)\|_{1} \leq K, \quad \forall t \geq 0
$$

where $K$ is the $\mathcal{V}_{1}$-radius of $\mathcal{B}_{1}$. Our aim is then to prove that there exist $\mathcal{R}_{2}=\mathcal{R}_{2}(K)$ and a positive constant $\alpha$ such that

$$
\operatorname{dist}_{1}\left(U(t),\left\{\xi \in \mathcal{V}_{2},\|\xi\|_{2} \leq \mathcal{R}_{2}\right\}\right) \leq C \mathrm{e}^{-\alpha t}
$$

Here and below $C$ is a generic positive constant allowed to depend on $K$ in (3.89).

In order to check (3.90), we will use a decomposition $U=V+W$ where the function $V=\left(v, v_{t}\right)$ is assumed to solve

$$
\varepsilon v_{t t}+v_{t}+A^{2} v-\operatorname{div}\left(f^{\prime}(u) \nabla v\right)+L v=g+L u, \quad V(0)=0,
$$


where $L=L(K)>0$ is a sufficiently large constant which will be fixed later. Then, the remainder $W=\left(w, w_{t}\right)$ will solve

$$
\varepsilon w_{t t}+w_{t}+A^{2} w-\operatorname{div}\left(f^{\prime}(u) \nabla w\right)+L w=0, \quad W(0)=U_{0} .
$$

We start by managing the equation for $w$.

LEMMA 3.5. There exists $L=L(K)$ such that

$$
\|W(t)\|_{1} \leq C \mathrm{e}^{-\alpha t}, \quad \forall t \geq 0,
$$

for some constants $C$ and $\alpha$ depending only on $K$.

Proof. Indeed, estimate (3.93) follows in a standard way by multiplying Eq. (3.92) by $w_{t}+\mu w$. The only nontrivial term $\left(f^{\prime \prime}(u) u_{t},|\nabla w|^{2}\right)$ can actually be estimated as follows

$$
\left|\left(f^{\prime \prime}(u) u_{t},|\nabla w|^{2}\right)\right| \leq C\left\|u_{t}\right\|\|\nabla w\|_{L^{4}(\Omega)}^{2} \leq \frac{1}{2}\|w\|_{H^{2}(\Omega)}^{2}+M_{K}\|w\|^{2},
$$

where $M_{K}$ is a positive constant depending only on $f$ and $K$ and whose value determines the choice of $L$.

We now study the component $V$ satisfying equation (3.91). To this end, we first note that, due to (3.89) and (3.93), it is already known that

$$
\|V(t)\|_{1} \leq C, \quad \forall t \geq 0 .
$$

The next lemma shows that this component belongs to $\mathcal{V}_{2}$ as well.

LEMMA 3.6. The solution $v(t)$ satisfies

$$
\|V(t)\|_{2} \leq C, \quad \forall t \geq 0 .
$$

Proof. Let $\Theta=\left(\theta, \theta_{t}\right):=V_{t}$. Then, multiplying Eq. (3.91) by $A v$, integrating over $\Omega$ and using (2.8) and (3.95), we see that

$$
\|v(t)\|_{H^{3}(\Omega)} \leq C\|\Theta(t)\|_{0} .
$$

Hence, we only need to estimate the $\mathcal{V}_{0}$-norm of the function $\Theta(t)$, where

$$
\varepsilon \theta_{t t}+\theta_{t}+A^{2} \theta-\operatorname{div}\left(f^{\prime}(u) \nabla \theta\right)-\operatorname{div}\left(f^{\prime \prime}(u) u_{t} \nabla v\right)+L \theta=L u_{t} .
$$

We now claim that multiplying this equation by $A^{-1}\left(\theta_{t}+\mu \theta\right)$ there follows

$$
\|\Theta(t)\|_{0} \leq C,
$$

which, thanks to (3.97), would finish the proof. To demonstrate our claim, we just estimate the most difficult terms coming from the nonlinearity $f$. Firstly, we have

$$
\begin{aligned}
\|\left\langle\operatorname{div}\left(f^{\prime \prime}(u) u_{t} \nabla v\right), A^{-1} \theta_{t}\right\rangle \mid & \leq \mu\left\|\theta_{t}\right\|_{V^{\prime}}^{2}+C_{\mu}\left\|f^{\prime \prime}(u)\right\|_{L^{\infty}(\Omega)}^{2}\left\|u_{t}\right\|^{2}\|\nabla v\|_{L^{\infty}(\Omega)}^{2} \\
& \leq \mu\left\|\theta_{t}\right\|_{V^{\prime}}^{2}+C_{\mu}\|\nabla v\|_{L^{\infty}(\Omega)}^{2} \\
& \leq \mu\|\Theta\|_{0}^{2}+C_{\mu} .
\end{aligned}
$$


Here we have used (3.89) and (3.97) and the well-known inequality

$$
\|\nabla v\|_{L^{\infty}(\Omega)}^{2} \leq c\|v\|_{H^{2}(\Omega)}\|v\|_{H^{3}(\Omega)} .
$$

Next, the first nonlinear term coming from (3.98) is transformed as follows:

$$
\begin{aligned}
-\left\langle\operatorname{div}\left(f^{\prime}(u) \nabla \theta\right), A^{-1} \theta_{t}\right\rangle= & \left(f^{\prime}(u) \theta, \theta_{t}\right)+\left\langle\operatorname{div}\left(f^{\prime \prime}(u) \theta \nabla u\right), A^{-1} \theta_{t}\right\rangle \\
= & \frac{1}{2} \frac{\mathrm{d}}{\mathrm{d} t}\left(f^{\prime}(u) \theta, \theta\right)-\frac{1}{2}\left(f^{\prime \prime}(u) u_{t},|\theta|^{2}\right) \\
& -\left(f^{\prime \prime}(u) \theta \nabla u, \nabla A^{-1} \theta_{t}\right) .
\end{aligned}
$$

Hence, we only need to estimate the last two terms in the right hand side above. The first is treated by noting that

$$
\left|\left(f^{\prime \prime}(u) u_{t},|\theta|^{2}\right)\right| \leq C\left\|u_{t}\right\|_{H}\|\theta\|_{L^{4}(\Omega)}^{2} \leq \mu\|\theta\|_{V}^{2}+C_{\mu},
$$

while for the second term we have

$$
\left|\left(f^{\prime \prime}(u) \theta \nabla u, \nabla A^{-1} \theta_{t}\right)\right| \leq \mu\left\|\theta_{t}\right\|_{V^{\prime}}^{2}+C_{\mu}\|\nabla u\|_{L^{6}(\Omega)}^{2}\|\theta\|_{L^{3}(\Omega)}^{2} \leq \mu\|\Theta\|_{0}^{2}+C_{\mu},
$$

where we have used that $\|\theta\|_{L^{3}(\Omega)}^{2} \leq c\|\theta\|_{H}\|\theta\|_{V}$. This concludes the proof of the claim and of the lemma.

We conclude by proving the $\mathcal{V}_{2}$-dissipativity.

LEMMA 3.7. Let the assumptions of Theorem 2.7 hold. Letting in addition $U_{0} \in \mathcal{V}_{2}$, the corresponding solution $U$ satisfies the estimate

$$
\|U(t)\|_{2} \leq Q\left(\left\|U_{0}\right\|_{2}\right) \mathrm{e}^{-\kappa t}+Q(\|g\|), \quad \forall t \geq 0 .
$$

Proof. Let us differentiate (2.14) with respect to time and $\operatorname{set} \theta:=u_{t}$ and $\Theta:=\left(\theta, \theta_{t}\right)$. We then obtain

$$
A^{-1}\left(\varepsilon \theta_{t t}+\theta_{t}\right)+A \theta+f^{\prime}(u) \theta=0 .
$$

We now test (3.106) by $2 \theta_{t}$. Of course, this procedure might be not justified in the current regularity setting, but could be made rigorous by working in the Galerkin approximation and then taking the limit. We omit the standard details. Simple computations lead to

$$
\frac{\mathrm{d}}{\mathrm{d} t}\left[\|\Theta\|_{0}^{2}+\left(f^{\prime}(u) \theta, \theta\right)\right]+2\left\|\theta_{t}\right\|_{V^{\prime}}^{2} \leq\left(f^{\prime \prime}(u) \theta^{2}, \theta\right) .
$$

Next, we test (3.106) by $\alpha \theta$, for $\alpha>0$ to be chosen. We get

$$
\frac{\mathrm{d}}{\mathrm{d} t}\left[\alpha \varepsilon\left(\theta_{t}, A^{-1} \theta\right)+\frac{\alpha}{2}\|\theta\|_{V^{\prime}}^{2}\right]-\alpha \varepsilon\left\|\theta_{t}\right\|_{V^{\prime}}^{2}+\alpha\|\theta\|_{V}^{2}+\alpha\left(f^{\prime}(u) \theta, \theta\right)=0 .
$$


Hence, using the $C^{2}$-regularity of $f$, Sobolev's embeddings and (2.24), we obtain

$$
\begin{aligned}
\left(f^{\prime \prime}(u) \theta^{2}, \theta\right) & \leq Q\left(\|u\|_{D(A)}\right)\|\theta\|^{3 / 2}\|\theta\|_{V}^{3 / 2} \\
& \leq \frac{\alpha}{2}\|\theta\|_{V}^{2}+Q\left(Q\left(\left\|U_{0}\right\|_{1}\right) \mathrm{e}^{-\kappa t}+Q(\|g\|)\right) .
\end{aligned}
$$

Thus, summing (3.107) and (3.108) together and exploiting (3.109) we obtain

$$
\frac{\mathrm{d}}{\mathrm{d} t} \Sigma+\kappa \Sigma \leq Q\left(Q\left(\left\|U_{0}\right\|_{1}\right) \mathrm{e}^{-\kappa t}+Q(\|g\|)\right),
$$

where we have taken $\alpha$ small enough and set

$$
\Sigma:=\|\Theta\|_{0}^{2}+\left(f^{\prime}(u) \theta, \theta\right)+\alpha \varepsilon\left(\theta_{t}, A^{-1} \theta\right)+\frac{\alpha}{2}\|\theta\|_{V}^{2}+Q\left(\left\|U_{0}\right\|_{1}\right) \mathrm{e}^{-\kappa t}+Q(\|g\|) .
$$

Here, on account of (2.8) and (2.24), $Q$ has been chosen such that

$$
c_{1}\|\Theta\|_{0}^{2} \leq \Sigma \leq c_{2}\|\Theta\|_{0}^{2}+Q\left(\left\|U_{0}\right\|_{1}\right) \mathrm{e}^{-\kappa t}+Q(\|g\|),
$$

for some $c_{1}, c_{2}>0$. Thus, proceeding as in (3.84) to control the right hand side of (3.110), we get a dissipative estimate for $\|\Theta\|_{0}^{2}$. Finally, let us prove (3.105). With this aim, let us first observe that, applying a comparison argument in (2.14), we get

$$
\begin{aligned}
\|U\|_{2} & \leq C\left(\|U\|_{1}+\|g\|\right)+C\|f(u)\|_{V} \\
& \leq C\left(\|U\|_{1}+\|g\|\right)+C\left\|f^{\prime}(u) \nabla u\right\|+C\|f(u)\| .
\end{aligned}
$$

Then, estimating the latter term by a further comparison of terms in (2.14) (and not just by using the regularity properties of $f$ ), the chain of inequalities is continued as follows

$$
\begin{aligned}
& \leq C\left(\|U\|_{1}+\|g\|\right)+C\left\|f^{\prime}(u)\right\|_{L^{\infty}(\Omega)}\|\nabla u\|+C\|U\|_{1} \\
& \leq\left(C+Q\left(\|U\|_{1}\right)\right)\left(\|U\|_{1}+\|g\|\right),
\end{aligned}
$$

where $Q$ is as usual a monotone function. Thus, the first factor in the last row is uniformly bounded, while the latter dissipates thanks to (2.24). The proof is concluded.

\subsection{Asymptotic boundedness in $\mathcal{V}_{3}$}

We conclude here the proof of Theorem 2.10 by showing that the semigroup $S_{\varepsilon}$ admits an exponentially attracting set $\mathcal{A}_{3}$ bounded in $\mathcal{V}_{3}$. To prove this, we start noting a simple fact, namely,

LEMMA 3.8. Let the assumptions of Theorem 2.7 hold. Suppose, in addition, that $f \in C_{\text {loc }}^{2,1}(\mathbb{R} ; \mathbb{R})$. Then $S_{\varepsilon}(t)$ is $\mathcal{V}_{1}$-Lipschitz continuous on any bounded positively invariant $\mathcal{P}_{1}$ sets of $\mathcal{V}_{1}$, with Lipschitz constant of the form $C_{1} e^{C_{2} t}, C_{1}$ and $C_{2}$ being positive constants depending only on the $\mathcal{V}_{1}$-radius of $\mathcal{P}_{1}$. 
Proof. Let us consider a couple of initial conditions $U_{0}=\left(u_{0}, u_{1}\right)$ and $V_{0}=\left(v_{0}, v_{1}\right)$ lying in a bounded and positively invariant subset $\mathcal{P}_{1}$ of $\mathcal{V}_{1}$. The, let us denote by $U=\left(u, u_{t}\right)$ and $V=\left(v, v_{t}\right)$ the two solutions to (2.14) originating, respectively, from $U_{0}$ and $V_{0}$. The function $W:=U-V$ will then solve

$$
A^{-1}\left(\varepsilon w_{t t}+w_{t}\right)+A w+f(u)-f(v)=0 .
$$

Then, multiplying (3.115) by $2 A w_{t}$ and integrating over $\Omega$, we get

$$
\varepsilon \frac{\mathrm{d}}{\mathrm{d} t}\left\|w_{t}\right\|^{2}+2\left\|w_{t}\right\|^{2}+\frac{\mathrm{d}}{\mathrm{d} t}\|A w\|^{2}=-2\left(A(f(u)-f(v)), w_{t}\right) .
$$

Using the positive invariance of $\mathcal{P}_{1}$, the fact that $f^{\prime \prime}$ is locally Lipschitz continuous, some standard Sobolev embeddings and the Young inequality, it is not difficult to see that the right hand side in (3.116) could be estimated as

$$
2\left(A(f(u)-f(v)), w_{t}\right) \leq C\|A w\|^{2}+\left\|w_{t}\right\|^{2},
$$

for $C$ depending on the $\mathcal{V}_{1}$-radius of $\mathcal{P}_{1}$. Thus, the Gronwall Lemma entails the desired (local) Lipschitz continuity of $S_{\varepsilon}(t)$.

We will now consider solutions $U=\left(u, u_{t}\right)$ whose initial datum $U_{0}$ lies in $\mathcal{A}_{2}$ and show that they are exponentially attracted with respect to the $\mathcal{V}_{1}$-norm (actually, also by the $\mathcal{V}_{2}$-norm) by a $\mathcal{V}_{3}$-bounded set $\mathcal{A}_{3}$. This will conclude the proof of Theorem 2.10 by means of the transitivity property of exponential attraction introduced in [10, Thm. 5.1]. Note that the Lipschitz condition [10, (5.1)] holds thanks to Lemma 3.8.

The construction of the attracting set $\mathcal{A}_{3}$ will follow the lines of Theorem 4.1 in [20] and is divided into a number of steps presented as separate Lemmas. First of all, we operate a new decomposition of a $U=\left(u, u_{t}\right)$ as a sum $U=V+W$, where $V=\left(v, v_{t}\right)$ and $W=\left(w, w_{t}\right)$, as usual. We take (a new) $L>0$ (whose value will be chosen later) and assume $V$ solves now

$$
A^{-1}\left(\varepsilon v_{t t}+v_{t}\right)+A v+L A^{-1} v+f(v)=A^{-1}(L u+g)=: A^{-1} h,\left.\quad V\right|_{t=0}=0,
$$

while $W$ is a solution of

$$
A^{-1}\left(\varepsilon w_{t t}+w_{t}\right)+A w+L A^{-1} w+f(u)-f(v)=0,\left.\quad W\right|_{t=0}=U_{0}:=\left(u_{0}, u_{1}\right),
$$

and we recall that $U_{0} \in \mathcal{V}_{2}$. We will prove that $V(\cdot)$ is compact in $\mathcal{V}_{2}$ (and, more precisely, bounded in $\mathcal{V}_{3}$ ), while $W(\cdot)$ exponentially decays in $\mathcal{V}_{2}$. We start with our first

LEMMA 3.9. Let the assumptions of Theorem 2.7 hold. Then, we have

$$
\|V(t)\|_{1} \leq Q_{L}\left(\left\|U_{0}\right\|_{1}\right) \mathrm{e}^{-\kappa t}+Q_{L}(\|g\|) .
$$


Proof. The proof relies on the same argument as the proof of (2.24) in Theorem 2.7. Thus, we limit ourselves to give the highlights. We namely introduce the parabolic problem

$$
A^{-1} v_{t}^{0}+A v^{0}+L A^{-1} v^{0}+f\left(v^{0}\right)=A^{-1}(L u+g)=A^{-1} h,\left.\quad v^{0}\right|_{t=0}=0,
$$

so that, setting $\bar{v}:=v-v_{0}$, we have (compare with (3.63))

$$
A^{-1} \bar{v}_{t}+A \bar{v}+L A^{-1} \bar{v}+f(v)-f\left(v^{0}\right)=-\varepsilon A^{-1} v_{t t},\left.\quad v^{0}\right|_{t=0}=0,
$$

Testing then (3.118) by $A\left(v_{t}+\alpha v\right)$ for small $\alpha>0$ and proceeding as in the proof of Theorem 2.7, we arrive at the analogue of (3.70), namely

$$
\frac{\mathrm{d}}{\mathrm{d} t} \mathcal{Z}(t)+\kappa \mathcal{Z}(t) \leq c\|h\|^{2}+\frac{1}{4}\left\|f^{\prime \prime}(v)\right\|_{L^{\infty}(\Omega)}^{2}\|v\|_{W^{1,4}(\Omega)}^{4},
$$

where this new $\mathcal{Z}$ is given by

$$
\mathcal{Z}(t):=\frac{\varepsilon}{2}\left\|v_{t}\right\|^{2}+\frac{1}{2}\|v\|_{D(A)}^{2}+\frac{L}{2}\|v\|^{2}+\frac{1}{2}\left(f^{\prime}(v) \nabla v, \nabla v\right)+\varepsilon \alpha\left(v_{t}, v\right) .
$$

Then, using $v^{0}$ in order to estimate the latter term on the right hand side of (3.123) and noting that

$$
\|h\|^{2} \leq Q_{L}\left(\left\|U_{0}\right\|_{1}\right) \mathrm{e}^{-\kappa t}+Q_{L}(\|g\|),
$$

we arrive at an expression analogous to (3.75), but related to the function $v$. Thus, we find that there exists a (new, and possibly smaller) number $\varepsilon_{0}>0$ such that, for $\varepsilon \in\left(0, \varepsilon_{0}\right)$, the analogue of (3.81) holds. Noting that no restriction is now required on the initial datum being in fact $V(0)=0$, we obtain (3.120). The proof is complete.

Let us now notice that, comparing (2.24) and (3.120), we obtain the analogue for the component $W$, namely

$$
\|W(t)\|_{1} \leq Q_{L}\left(\left\|U_{0}\right\|_{1}\right) \mathrm{e}^{-\kappa t}+Q_{L}(\|g\|) .
$$

We now prove that our system admits a dissipation integral in the $V$-norm.

LEMMA 3.10. Let the assumptions of Theorem 2.7 hold. Then, for any quasi-strong solution $U=\left(u, u_{t}\right)$ to $P_{\varepsilon}$ there holds

$$
\int_{0}^{+\infty}\left\|u_{t}(s)\right\|_{V}^{2} \mathrm{~d} s+\sup _{s \in[0,+\infty)} \varepsilon\left\|u_{t t}(s)\right\|_{V^{\prime}}^{2} \leq Q\left(\left\|U_{0}\right\|_{2}\right)<\infty .
$$

Proof. Let us differentiate once more (2.14) with respect to time and set $\theta:=u_{t}$ and $\Theta:=\left(\theta, \theta_{t}\right)$. We then get

$$
A^{-1}\left(\varepsilon \theta_{t t}+\theta_{t}\right)+A \theta+L A^{-1} \theta+f^{\prime}(u) \theta=L A^{-1} u_{t}=: k .
$$


We now test (3.128) by $2 \theta_{t}$. Then, simple computations lead to

$$
\begin{aligned}
& \frac{\mathrm{d}}{\mathrm{d} t}\left[\|\Theta\|_{0}^{2}+L\|\theta\|_{V^{\prime}}^{2}+\left(f^{\prime}(u) \theta, \theta\right)\right]+\left\|\theta_{t}\right\|_{V^{\prime}}^{2} \\
& \quad \leq\|k\|_{V}^{2}+\left(f^{\prime \prime}(u) \theta^{2}, \theta\right) \leq\|k\|_{V}^{2}+Q\left(\|U\|_{1}\right)\|\theta\|_{L^{3}(\Omega)}^{3},
\end{aligned}
$$

where $Q$ can depend on $\varepsilon$, but is independent of $L$. Let us now observe that

$$
Q\left(\|U\|_{1}\right)\|\theta\|_{L^{3}(\Omega)}^{3} \leq Q\left(\|U\|_{1}\right)\|\theta\|_{V}^{9 / 4}\|\theta\|_{V^{\prime}}^{3 / 4} \leq Q_{9}\left(\|U\|_{2}\right)\|\theta\|_{V^{\prime}}^{2}+\frac{1}{8}\|\theta\|_{V}^{2} .
$$

Next, we test (3.128) by $\theta / 2$, inferring

$$
\begin{aligned}
& \frac{\mathrm{d}}{\mathrm{d} t}\left[\frac{\varepsilon}{2}\left(\theta_{t}, A^{-1} \theta\right)+\frac{1}{4}\|\theta\|_{V^{\prime}}^{2}\right]-\frac{\varepsilon}{2}\left\|\theta_{t}\right\|_{V^{\prime}}^{2}+\frac{1}{2}\|\theta\|_{V}^{2}+\frac{L}{2}\|\theta\|_{V^{\prime}}^{2}+\frac{1}{2}\left(f^{\prime}(u) \theta, \theta\right) \\
& \quad=\frac{1}{2}(k, \theta) \leq \frac{1}{8}\|\theta\|_{V}^{2}+c\|k\|_{V}^{2} .
\end{aligned}
$$

Summing (3.129) and (3.131), we then get

$$
\begin{aligned}
& \frac{\mathrm{d}}{\mathrm{d} t}\left[\|\Theta\|_{0}^{2}+\left(L+\frac{1}{4}\right)\|\theta\|_{V^{\prime}}^{2}+\left(f^{\prime}(u) \theta, \theta\right)+\frac{\varepsilon}{2}\left(\theta_{t}, A^{-1} \theta\right)\right] \\
& \quad+\left(1-\frac{\varepsilon}{2}\right)\left\|\theta_{t}\right\|_{V^{\prime}}^{2}+\frac{1}{4}\|\theta\|_{V}^{2}+\frac{L-2 Q_{9}}{2}\|\theta\|_{V^{\prime}}^{2}+\frac{1}{2}\left(f^{\prime}(u) \theta, \theta\right) \leq c\|k\|_{V}^{2},
\end{aligned}
$$

still for $C$ independent of $L$. Here $Q_{9}$ denotes the quantity $Q_{9}\left(\|U\|_{2}\right)$ on the right hand side of (3.130). Thus, noting as a (new) functional $\mathcal{Y}$ the quantity in square brackets, we observe that we can choose $L$ so large (depending on $\lambda$ in (2.8) and on $Q_{9}$, i.e., on the norms of the initial datum and of $g$ ) to get

$$
\frac{\mathrm{d}}{\mathrm{d} t} \mathcal{Y}+\kappa \mathcal{Y} \leq c\|k\|_{V}^{2}
$$

for some $\kappa>0$, where $\mathcal{Y}$ satisfies

$$
C_{L}\|\Theta\|_{0}^{2} \leq \mathcal{Y} \leq c_{L, \varepsilon}\|\Theta\|_{0}^{2}+Q_{L}\left(\|U\|_{1}\right)
$$

Hence, recalling that $k=L A^{-1} u_{t}$ and using (3.9) and the fact that $\mathcal{Y}(0)=Q\left(\left\|U_{0}\right\|_{2}\right)$, (3.127) follows immediately.

We now prove the exponential decay of $W$ in the $\mathcal{V}_{0}$-norm.

LEMMA 3.11. Let the assumptions of Theorem 2.7 hold. Then, L can be chosen so large that

$$
\|W(t)\|_{0} \leq Q_{L}\left(\left\|U_{0}\right\|_{2}\right) \mathrm{e}^{-\kappa t}
$$


Proof. We proceed along the lines of the preceding proof. First, we test (3.119) by $2 w_{t}$, so that

$$
\frac{\mathrm{d}}{\mathrm{d} t}\left[\|W\|_{0}^{2}+L\|w\|_{V^{\prime}}^{2}+2 \mathcal{I}_{1}\right]+\left\|w_{t}\right\|_{V^{\prime}}^{2} \leq 2 \mathcal{I}_{2},
$$

where $\mathcal{I}_{1}$ and $\mathcal{I}_{2}$ collect the terms coming from $f$. Namely, we have (recall that $U=V+W)$

$$
\mathcal{I}_{1}=(F(u-w)-F(u)+f(u) w, 1) \geq-\frac{\lambda}{2}\|w\|^{2}
$$

thanks to (2.8). To estimate $\mathcal{I}_{2}$, let us first notice that, performing the standard energy estimate on (3.118) (i.e., testing it by $v_{t}+\alpha v$ for small $\alpha>0$ ) and using the energy estimate (2.25) for $u$ to control the term on the right hand side, we derive

$$
\|V(t)\|_{0}^{2} \leq Q_{L}\left(\left\|U_{0}\right\|_{1}\right) \mathrm{e}^{-\kappa t}+C_{L},
$$

where both $C_{L}$ and $Q_{L}$ depend on $L$ since so does the right hand side of (3.118). Comparing (2.25) and (3.137), we also get

$$
\|W(t)\|_{0}^{2} \leq Q_{L}\left(\left\|U_{0}\right\|_{1}\right) \mathrm{e}^{-\kappa t}+C_{L} .
$$

Using now the uniform $\mathcal{V}_{2}$-bound on $U,(3.138)$, and standard interpolation and embeddings, we can estimate

$$
\begin{aligned}
\mathcal{I}_{2}= & \left(f(u-w)-f(u)+f^{\prime}(u) w, u_{t}\right) \leq Q\left(\|u\|_{L^{\infty}(\Omega)}\right. \\
& \left.+\|w\|_{L^{\infty}(\Omega)}\right)\left(|w|^{2}\left|u_{t}\right|, 1\right) \\
\leq & Q\left(\|u\|_{D(A)}+\|w\|_{D(A)}\right)\left\|u_{t}\right\|_{V}\|w\|_{V}^{2} \\
\leq & \frac{1}{16}\|w\|_{V}^{2}+Q_{L}\left\|u_{t}\right\|_{V}^{2}\|w\|_{V}^{2}
\end{aligned}
$$

where we used the fact that the $D(A)$-norms of $u$ and $w$ are uniformly bounded thanks, respectively, to (2.24) and (3.126), in a way which depends just on the $\mathcal{V}_{2}$-norm of $U_{0}$. Actually, $Q_{L}$ denotes here a quantity which depends on the $\mathcal{V}_{2}$-norm of $U_{0}$ and on $L$. Next, we test (3.119) by $w / 2$, inferring

$$
\begin{aligned}
& \frac{\mathrm{d}}{\mathrm{d} t}\left[\frac{\varepsilon}{2}\left(w_{t}, A^{-1} w\right)+\frac{1}{4}\|w\|_{V^{\prime}}^{2}\right]-\frac{\varepsilon}{2}\left\|w_{t}\right\|_{V^{\prime}}^{2}+\frac{1}{2}\|w\|_{V}^{2}+\frac{L}{2}\|w\|_{V^{\prime}}^{2} \\
& \quad+\frac{1}{2}(f(u)-f(u-w), w)=0 .
\end{aligned}
$$

Now, using (2.8) and interpolation, it is not difficult to compute

$$
\begin{aligned}
(f(u)-f(u-w), w)= & (f(u)-f(u-w), w)+(F(u-w)-F(u), 1) \\
& -(F(u-w)-F(u), 1) \\
= & \mathcal{I}_{1}+(F(u)-F(u-w)-f(u-w) w, 1) \\
\geq & \mathcal{I}_{1}-\frac{\lambda}{2}\|w\|^{2} \geq \mathcal{I}_{1}-\frac{1}{4}\|w\|_{V}^{2}-C\|w\|_{V^{\prime}}^{2},
\end{aligned}
$$


for some $C>0$ possibly depending also on the initial datum. Thus, adding (3.135) and (3.140), we arrive at

$$
\begin{aligned}
& \frac{\mathrm{d}}{\mathrm{d} t}\left[\|W\|_{0}^{2}+\left(L+\frac{1}{4}\right)\|w\|_{V^{\prime}}^{2}+2 \mathcal{I}_{1}+\frac{\varepsilon}{2}\left(w_{t}, A^{-1} w\right)\right] \\
& \quad+\left(1-\frac{\varepsilon}{2}\right)\left\|w_{t}\right\|_{V^{\prime}}^{2}+\frac{1}{4}\|w\|_{V}^{2}+\frac{L-C}{2}\|w\|_{V^{\prime}}^{2}+\frac{1}{2} \mathcal{I}_{1} \leq Q_{L}\left\|u_{t}\right\|_{V}^{2}\|w\|_{V}^{2} .
\end{aligned}
$$

Finally, possibly choosing a larger $L$ such that

$$
L \geq 2 C \text { and } \frac{L}{2}\|w\|_{V^{\prime}}^{2}+\mathcal{I}_{1}+\frac{1}{8}\|w\|_{V}^{2} \geq 0,
$$

we can rewrite $(3.142)$ in the form

$$
\frac{\mathrm{d}}{\mathrm{d} t} \mathcal{Y}+\kappa \mathcal{Y} \leq m \mathcal{Y}
$$

where $\mathcal{Y}$ denotes once more the quantity in square brackets and (cf. (3.127))

$$
m:=Q_{L}\left\|u_{t}\right\|_{V}^{2} \in L^{1}(0,+\infty)
$$

Thus, the comparison principle for ODEs readily gives (3.134).

Next, we can prove a $\mathcal{V}_{2}$-dissipativity estimate for (3.118). Of course, when (3.118) is differentiated with respect to time, the "source" term $L A^{-1} u_{t}$ in the right hand side is easily controlled thanks to (2.25). Hence, we get the estimate

$$
\|V(t)\|_{2}^{2}+\left\|v_{t t}(t)\right\|_{V^{\prime}}^{2} \leq Q\left(\left\|U_{0}\right\|_{2}\right) \mathrm{e}^{-\kappa t}+Q\left(\|g\|_{V^{\prime}}\right),
$$

where we stress that from now on the dependence on $L$ of the constants and the functions $Q$ is no longer indicated.

As a next step, we prove that the component $V$ of the solution is compact in $\mathcal{V}_{2}$ and, more precisely, bounded in $\mathcal{V}_{3}$.

LEMMA 3.12. Let the assumptions of Lemma 3.8 hold (hence, in particular, let $\left.f \in C_{\text {loc }}^{2,1}(\mathbb{R} ; \mathbb{R})\right)$. Then we have

$$
\|V(t)\|_{3} \leq Q\left(\left\|U_{0}\right\|_{2}\right) \mathrm{e}^{-\kappa t}+Q(\|g\|) .
$$

Proof. We differentiate (3.118) in time and test the result by $A\left(v_{t t}+\alpha v_{t}\right)$ for small $\alpha>0$. We do not give all the details, but just see how the nonlinear terms are controlled. Actually, performing some calculation and using (3.147) and interpolation, we get

$$
\begin{aligned}
\left(f^{\prime}(v) v_{t}, A v_{t t}\right)= & \frac{1}{2} \frac{\mathrm{d}}{\mathrm{d} t}\left(f^{\prime}(v)\left|\nabla v_{t}\right|^{2}, 1\right)-\frac{1}{2}\left(f^{\prime \prime}(v) v_{t}\left|\nabla v_{t}\right|^{2}, 1\right) \\
& -\left(\operatorname{div}\left(f^{\prime \prime}(v) v_{t} \nabla v\right), v_{t t}\right) \\
\geq & \frac{1}{2} \frac{\mathrm{d}}{\mathrm{d} t}\left(f^{\prime}(v)\left|\nabla v_{t}\right|^{2}, 1\right)-\frac{\alpha}{4}\left\|v_{t}\right\|_{D(A)}^{2}-\frac{\varepsilon}{4}\left\|v_{t t}\right\|^{2}-C_{\alpha}\left\|v_{t}\right\|_{V}^{2},
\end{aligned}
$$


and, analogously,

$$
\alpha\left(f^{\prime}(v) v_{t}, A v_{t}\right) \geq \alpha \int_{\Omega} f^{\prime}(v)\left|\nabla v_{t}\right|^{2}-C\left\|v_{t}\right\|_{V}^{2},
$$

where in both formulas $C$ (or $C_{\alpha}$ ) is a monotone function of $\|V(t)\|_{2}$. Thus, noting that the right hand side term $L\left(u_{t}, v_{t t}+\delta v_{t}\right)$ can be estimated in a standard way, one arrives at an expression of the form

$$
\frac{\mathrm{d}}{\mathrm{d} t} \mathcal{Y}_{3}+\kappa \mathcal{Y}_{3} \leq Q\left(\|V(t)\|_{2}\right)+Q\left(\|U(t)\|_{2}\right) \leq Q\left(Q\left(\left\|U_{0}\right\|_{2}\right) e^{-\kappa t}+Q(\|g\|)\right),
$$

where Lemma 3.7 has been used to deduce the latter inequality, and the functional $\mathcal{Y}_{3}$ (upon possibly taking a larger $L$ ) satisfies

$$
c\left\|V_{t}\right\|_{1}^{2} \leq \mathcal{Y}_{3} \leq C\left\|V_{t}\right\|_{1}^{2}+Q\left(\|V\|_{2}\right),
$$

where $C$ depends on the radius of the absorbing set. Hence, we have a dissipativity estimate for $\left\|V_{t}\right\|_{1}^{2}$. At this point, we can deduce also the dissipativity of $\|V\|_{3}^{2}$, by performing a comparison argument perfectly analogous to the one used in the last part of the proof of Lemma 3.7 (and hence omitted). The proof is complete.

Finally, we show that $W$ is exponentially decaying in $\mathcal{V}_{2}$. Of course, this fact, together with (3.147), will give the desired property of the decomposition (3.118) and (3.119) and conclude the proof of Theorem 2.10.

LEMMA 3.13. Let the assumptions of Theorem 2.7 hold. Then we have

$$
\|W(t)\|_{2} \leq Q\left(\left\|U_{0}\right\|_{2}\right) \mathrm{e}^{-\kappa t} .
$$

Proof. We differentiate (3.119) in time and test the result by $w_{t t}+\alpha w_{t}$ for small $\alpha>0$. Still, the procedure is standard, but for the estimation of the nonlinear terms depending on $f$. Namely, we obtain on the left hand side

$$
\left((f(u)-f(u-w))_{t}, w_{t t}+\alpha w_{t}\right) .
$$

Thus, defining

$$
l=l(u, w):=\int_{0}^{1} f^{\prime}(s u+(1-s)(u-w)) \mathrm{d} s \geq-\lambda,
$$

so that $f(u)-f(u-w)=l w$, we clearly have

$$
\left((f(u)-f(u-w))_{t}, w_{t t}+\alpha w_{t}\right)=\left(l_{t} w+l w_{t}, w_{t t}+\alpha w_{t}\right)
$$

and

$$
\begin{aligned}
\left|\left(l_{t} w, w_{t t}+\alpha w_{t}\right)\right| & \leq\left\|l_{t} w\right\|_{V}\left\|w_{t t}+\alpha w_{t}\right\|_{V^{\prime}} \\
& \leq c\left(\left\|l_{t}\right\|_{V}\|w\|_{D(A)}\right)\left\|w_{t t}+\alpha w_{t}\right\|_{V^{\prime}}
\end{aligned}
$$


Now, let us notice that, by Lemma 3.7 and (3.146), there follows

$$
\|W(t)\|_{2} \leq Q\left(\left\|U_{0}\right\|_{2}\right) \mathrm{e}^{-\kappa t}+Q\left(\|g\|_{V^{\prime}}\right) .
$$

In particular, $\left\|l_{t}\right\|_{V} \leq C$, with $C$ possibly depending on $U_{0}$, but independent of time. More precisely, using (3.134) and interpolation, we get, for all $v>0$,

$$
\|W(t)\|_{2-v} \leq Q\left(\left\|U_{0}\right\|_{2}\right) \mathrm{e}^{-\kappa t},
$$

$\kappa$ depending here on $v$. Consequently (take $v=1$ ), we can control the right hand side of (3.156) so that

$$
\left|\left(l_{t} w, w_{t t}+\alpha w_{t}\right)\right| \leq Q\left(\left\|U_{0}\right\|_{2}\right) \mathrm{e}^{-\kappa t}+\frac{\varepsilon}{4}\left\|w_{t t}\right\|^{2}+\frac{\alpha}{4}\left\|w_{t}\right\|_{V^{\prime}}^{2},
$$

and the latter two terms can be moved to the left hand side and estimated directly. Finally, coming back to the remaining term in (3.155), we get

$$
\left(l w_{t}, w_{t t}+\alpha w_{t}\right)=\frac{1}{2} \frac{\mathrm{d}}{\mathrm{d} t}\left(l, w_{t}^{2}\right)+\alpha\left(l, w_{t}^{2}\right)-\frac{1}{2}\left(l_{t}, w_{t}^{2}\right),
$$

and the first two summands on the right hand side are controlled once more thanks to (2.8), while the third is estimated for small $v>0$ by

$$
-\frac{1}{2}\left(l_{t}, w_{t}^{2}\right) \leq c\left\|l_{t}\right\|_{V}\left\|w_{t}\right\|_{D\left(A^{\frac{1-v}{2}}\right.}^{2} \leq C\left\|w_{t}\right\|_{D\left(A^{\frac{1-v}{2}}\right)}^{2} \leq Q\left(\left\|U_{0}\right\|_{2}\right) \mathrm{e}^{-\kappa t},
$$

thanks to (3.158). Thus, all the nonlinear terms are either (essentially) positive, or exponentially decaying. Then, (3.152) is proven, which concludes the proof of Lemma 3.13 and of Theorem 2.10.

\section{Acknowledgments}

The authors thank the anonymous referee for her/his careful reading of the manuscript. The first three authors were partially supported by the Italian PRIN 2006 "Problemi a frontiera libera, transizioni di fase e modelli di isteresi".

\section{REFERENCES}

[1] A. Babin and M.I. Vishik, Maximal attractors of semigroups corresponding to evolutionary differential equations, Mat. Sb., 126 (1984), 397-419.

[2] J.M. Ball, Global attractors for damped semilinear wave equations. Partial differential equations and applications, Discrete Contin. Dyn. Syst., 10 (2004), 31-52.

[3] H. Brézis and T. Gallouet, Nonlinear Schrödinger evolution equations, Nonlinear Anal., 4 (1980), $677-681$.

[4] J.W. Cahn, On spinodal decomposition, Acta Metall., 9 (1961), 795-801.

[5] J.W. Cahn and J.E. Hilliard, Free energy of a nonuniform system. I. Interfacial free energy, J. Chem. Phys., 28 (1958), 258-267. 
[6] M. Conti and G. Mola, 3-D viscous Cahn-Hilliard with memory, Math. Methods Appl. Sci., (2008), doi:10.1002/mma.1091.

[7] A. Debussche, A singular perturbation of the Cahn-Hilliard equation, Asymptotic Anal., 4 (1991), $161-185$.

[8] M. Efendiev, A. Miranville, and S. Zelik, Exponential attractors for a singularly perturbed Cahn-Hilliard system, Math. Nachr., 272 (2004), 11-31.

[9] C.M. Elliott and S. Zheng, On the Cahn-Hilliard equation, Arch. Rational Mech. Anal., 96 (1986), 339-357.

[10] P. Fabrie, C. Galusinski, A. Miranville, and A. Zelik, Uniform exponential attractors for a singularly perturbed damped wave equation, in "Partial differential equations and applications", Discrete Contin. Dyn. Syst., 10 (2004), 211-238.

[11] P. Galenko and D. Jou, Diffuse-interface model for rapid phase transformations in nonequilibrium systems, Phys. Rev. E, 71 (2005), 046125 (13 pages).

[12] P. Galenko and V. Lebedev, Analysis of the dispersion relation in spinodal decomposition of a binary system, Philos. Mag. Lett., 87 (2007), 821-827.

[13] P. Galenko and V. Lebedev, Local nonequilibrium effect on spinodal decomposition in a binary system, Int. J. Thermodyn., 11 (2008), 21-28.

[14] P. Galenko and V. Lebedev, Nonequilibrium effects in spinodal decomposition of a binary system, Phys. Lett. A, 372 (2008), 985-989.

[15] S. Gatti, M. Grasselli, A. Miranville, and V. Pata, On the hyperbolic relaxation of the onedimensional Cahn-Hilliard equation, J. Math. Anal. Appl., 312 (2005), 230-247.

[16] S. Gatti, M. Grasselli, A. Miranville, and V. Pata, Hyperbolic relaxation of the viscous Cahn-Hilliard equation in 3-D, Math. Models Methods Appl. Sci., 15 (2005), 165-198.

[17] S. Gatti, M. Grasselli, A. Miranville, and V. Pata, Memory relaxation of the one-dimensional Cahn-Hilliard equation, Dissipative phase transitions, 101-114, Ser. Adv. Math. Appl. Sci., 71, World Sci. Publ., Hackensack, NJ, 2006.

[18] C.P. Grant, Spinodal decomposition for the Cahn-Hilliard equation, Comm. Partial Differential Equations, 18 (1993), 453-490.

[19] M. Grasselli, A. Miranville, V. Pata, and S. Zelik, Well-posedness and long time behavior of a parabolic-hyperbolic phase-field system with singular potential, Math. Nachr., 280 (2007), 1475-1509.

[20] M. Grasselli, G. Schimperna, and S. Zelik, On the 2D Cahn-Hilliard equation with inertial term, Comm. Partial Differential Equation, 34 (2009), 137-170.

[21] M.B. Kania, Global attractor for the perturbed viscous Cahn-Hilliard equation, Colloq. Math., 109 (2007), 217-229.

[22] N. Kenmochi, M. Niezgódka, and I. Pawłow, Subdifferential operator approach to the CahnHilliard equation with constraint, J. Differential Equations, 117 (1995), 320-356.

[23] S. Maier-Paape and T. Wanner, Spinodal decomposition for the Cahn-Hilliard equation in higher dimensions: nonlinear dynamics, Arch. Ration. Mech. Anal., 151 (2000), 187-219.

[24] A. Miranville and S. Zelik, Robust exponential attractors for Cahn-Hilliard type equations with singular potentials, Math. Methods Appl. Sci., 27 (2004), 545-582.

[25] A. Novick-Cohen and L.A. Segel, Nonlinear aspects of the Cahn-Hilliard equation, Phys. D, 10 (1984), 277-298.

[26] A. Novick-Cohen, The Cahn-Hilliard equation: mathematical and modeling perspectives, Adv. Math. Sci. Appl., 8 (1998), 965-985.

[27] A. Novick-Cohen, On Cahn-Hilliard type equations, Nonlinear Anal., 15 (1990), 797-814.

[28] B. Nicolaenko, B. Scheurer, and R. Temam, Some global dynamical properties of a class of pattern formation equations, Comm. Partial Differential Equations, 14 (1989), 245-297.

[29] V. Pata, G. Prouse, and M. I. Vishik, Traveling waves of dissipative nonautonomous hyperbolic equations in a strip, Adv. Differential Equations, 3 (1998), 249-270.

[30] V. Pata and S. Zelik, A result on the existence of global attractors for semigroups of closed operators, Commun. Pure Appl. Anal. 6 (2007), 481-486.

[31] P. Rybka and K.-H. Hoffmann, Convergence of solutions to Cahn-Hilliard equation, Comm. Partial Differential Equations, 24 (1999), 1055-1077.

[32] A. Segatti, On the hyperbolic relaxation of the Cahn-Hilliard equation in 3-D: approximation and long time behaviour, Math. Models Methods Appl. Sci., 17 (2007), 411-437. 
[33] R. Temam, "Infinite-Dimensional Dynamical Systems in Mechanics and Physics". Springer-Verlag, New York, 1997.

[34] V. Vergara, A conserved phase field system with memory and relaxed chemical potential, J. Math. Anal. Appl., 328 (2007), 789-812.

[35] W. von Wahl, On the Cahn-Hilliard equation $u^{\prime}+\Delta^{2} u-\Delta f(u)=0$, in Mathematics and mathematical engineering (Delft, 1985), Delft Progr. Rep., 10 (1985), 291-310.

[36] S. Zheng and A.J. Milani, Global attractors for singular perturbations of the Cahn-Hilliard equations, J. Differential Equations, 209 (2005), 101-139.

[37] S. Zheng and A.J. Milani, Exponential attractors and inertial manifolds for singular perturbations of the Cahn-Hilliard equations, Nonlinear Anal., 57 (2004), 843-877.

[38] S. Zelik, Asymptotic regularity of singularly perturbed damped wave equations with supercritical nonlinearities, Discrete Contin. Dyn. Syst., 11 (2004), 351-392.

[39] S. Zelik, Global averaging and parametric resonances in damped semilinear wave equations, Proc. Roy. Soc. Edinburgh Sect. A, 136 (2006), 1053-1097.

M. Grasselli

Dipartimento di Matematica, Politecnico di Milano,

Via Bonardi 9, 20133 Milan, Italy

E-mail: maurizio.grasselli@polimi.it

G. Schimperna and A. Segatti Dipartimento di Matematica, Università di Pavia,

Via Ferrata 1, 27100 Pavia, Italy

E-mail: giusch04@unipv.it, antonio.segatti@unipv.it

S. Zelik

Department of Mathematics, University of Surrey,

Guildford GU2 7XH, UK

E-mail: S.Zelik@surrey.ac.uk 